\title{
A Novel Method for the Synthesis of Dinucleoside Boranophosphates by a Boranophosphotriester Method
}

\author{
Mamoru Shimizu, Takeshi Wada,* Natsuhisa Oka, and Kazuhiko Saigo \\ Departmet of Integrated Biosciences, Graduate School of Frontier Sciences, \\ The University of Tokyo \\ Bioscience Bldg. 702, Kashiwanoha, Kashiwa 277-8562, Japan
}

\section{Supporting Information}




\section{Table of contents}

General Information

Synthesis and analytical data of $O^{6}$-diphenylcarbamoyl- $N^{2}$-phenylacetyl-2'-deoxyguanosine

Synthesis and analytical data of $\mathbf{1}\left(\mathrm{B}^{1}=O^{6}\right.$-diphenylcarbamoyl- $N^{2}$-phenylacetylguanin-9-yl)

Analytical data of $\mathbf{3}\left(\mathrm{B}^{1}=N^{4}\right.$-benzoylcytosin-1-yl $)$

Analytical data of $19\left(\mathrm{~B}^{1}=O^{6}\right.$-diphenylcarbamoyl- $N^{2}$-phenylacetylguanin-9-yl)

Analytical data of $4\left(\mathrm{~B}^{1}=N^{4}\right.$-benzoylcytosin-1-yl $)$

Analytical data of $\mathbf{2 0}\left(\mathrm{B}^{1}=O^{6}\right.$-diphenylcarbamoyl- $N^{2}$-phenylacetylguanin-9-yl $)$

Analytical data of $6\left(\mathrm{~B}^{1}=N^{4}\right.$-benzoylcytosin-1-yl, $\mathrm{B}^{2}=N^{3}$-benzoylthymin-1-yl $)$

Analytical data of $6\left(\mathrm{~B}^{1}=O^{6}\right.$-diphenylcarbamoyl- $N^{2}$-phenylacetylguanin-9-yl,

$\mathrm{B}^{2}=N^{3}$-benzoylthymin-1-yl)

Analytical data of $6\left(\mathrm{~B}^{1}=\mathrm{B}^{2}=N^{3}\right.$-benzoylthymin-1-yl $)$

Analytical data of $\mathbf{6}\left(\mathrm{B}^{1}=N^{3}\right.$-benzoylthymin-1-yl, $\mathrm{B}^{2}=N^{4}$-benzoylcytosin-1-yl $)$

Analytical data of $6\left(\mathrm{~B}^{1}=N^{3}\right.$-benzoylthymin-1-yl,

$\mathrm{B}^{2}=N^{6}$-diphenylcarbamoyl- $N^{2}$-phenylacetylguanin-9-yl)

Analytical data of $27\left(B^{1}=\right.$ adenin-9-yl, $B^{2}=$ thymin-1-yl $)$

Analytical data of $27\left(\mathrm{~B}^{1}=\right.$ cytosin-1-yl, $\mathrm{B}^{2}=$ thymin-1-yl $)$

${ }^{1} \mathrm{H}$ and ${ }^{13} \mathrm{C}$ NMR of $O^{6}$-diphenylcarbamoyl- $N^{2}$-phenylacetyl-2'-deoxyguanosine

${ }^{1} \mathrm{H}$ and ${ }^{13} \mathrm{C}$ NMR of $1\left(\mathrm{~B}^{1}=O^{6}\right.$-diphenylcarbamoyl- $N^{2}$-phenylacetylguanin-9-yl)

${ }^{1} \mathrm{H}$ and ${ }^{13} \mathrm{C} \mathrm{NMR}$ of 2

${ }^{1} \mathrm{H},{ }^{13} \mathrm{C}$ and ${ }^{31} \mathrm{P}$ NMR of 17

${ }^{1} \mathrm{H},{ }^{13} \mathrm{C}$ and ${ }^{31} \mathrm{P}$ NMR of $\mathbf{3}\left(\mathrm{B}^{1}=N^{6}\right.$-benzoyladenin-9-yl $)$ 
${ }^{1} \mathrm{H},{ }^{13} \mathrm{C}$ and ${ }^{31} \mathrm{P}$ NMR of $\mathbf{3}\left(\mathrm{B}^{1}=N^{4}\right.$-benzoylcytosin-1-yl) 18

${ }^{1} \mathrm{H},{ }^{13} \mathrm{C}$ and ${ }^{31} \mathrm{P}$ NMR of $\mathbf{1 9}\left(\mathrm{B}^{1}=N^{3}\right.$-benzoylthymin-1-yl) 19

${ }^{1} \mathrm{H}$ and ${ }^{31} \mathrm{P}$ NMR of $19\left(\mathrm{~B}^{1}=O^{6}\right.$-diphenylcarbamoyl- $N^{2}$-phenylacetylguanin-9-yl $) \quad 20$

${ }^{1} \mathrm{H},{ }^{13} \mathrm{C}$ and ${ }^{31} \mathrm{P}$ NMR of $\mathbf{4}\left(\mathrm{B}^{1}=N^{6}\right.$-benzoyladenin-9-yl) 21

${ }^{1} \mathrm{H},{ }^{13} \mathrm{C}$ and ${ }^{31} \mathrm{P}$ NMR of $\mathbf{4}\left(\mathrm{B}^{1}=N^{4}\right.$-benzoylcytosin-1-yl) 22

${ }^{1} \mathrm{H},{ }^{13} \mathrm{C}$ and ${ }^{31} \mathrm{P}$ NMR of $20\left(\mathrm{~B}^{1}=O^{6}\right.$-diphenylcarbamoyl- $N^{2}$-phenylacetylguanin-9-yl) 23

${ }^{1} \mathrm{H},{ }^{13} \mathrm{C}$ and ${ }^{31} \mathrm{P}$ NMR of $20\left(\mathrm{~B}^{1}=N^{3}\right.$-benzoylthymin-1-yl) 24

${ }^{1} \mathrm{H},{ }^{13} \mathrm{C}$ and ${ }^{31} \mathrm{P}$ NMR of $6\left(\mathrm{~B}^{1}=N^{6}\right.$-benzoyladenin-9-yl, $\mathrm{B}^{2}=N^{3}$-benzoylthymin-1-yl $) \quad 25$

${ }^{1} \mathrm{H},{ }^{13} \mathrm{C}$ and ${ }^{31} \mathrm{P}$ NMR of $6\left(\mathrm{~B}^{1}=N^{4}\right.$-benzoylcytosin-1-yl, $\mathrm{B}^{2}=N^{3}$-benzoylthymin-1-yl $) 26$

${ }^{1} \mathrm{H},{ }^{13} \mathrm{C}$ and ${ }^{31} \mathrm{P}$ NMR of $6\left(\mathrm{~B}^{1}=O^{6}\right.$-diphenylcarbamoyl- $N^{2}$-phenylacetylguanin-9-yl,

$\mathrm{B}^{2}=N^{3}$-benzoylthymin-1-yl)

${ }^{1} \mathrm{H},{ }^{13} \mathrm{C}$ and ${ }^{31} \mathrm{P}$ NMR of $6\left(\mathrm{~B}^{1}=\mathrm{B}^{2}=N^{3}\right.$-benzoylthymin-1-yl) 28

${ }^{1} \mathrm{H},{ }^{13} \mathrm{C}$ and ${ }^{31} \mathrm{P}$ NMR of $6\left(\mathrm{~B}^{1}=N^{3}\right.$-benzoylthymin-1-yl, $\mathrm{B}^{2}=N^{6}$-benzoyladenin-9-yl $)$

${ }^{1} \mathrm{H},{ }^{13} \mathrm{C}$ and ${ }^{31} \mathrm{P}$ NMR of $6\left(\mathrm{~B}^{1}=N^{3}\right.$-benzoylthymin-1-yl, $\mathrm{B}^{2}=N^{4}$-benzoylcytosin-1-yl) 30

${ }^{1} \mathrm{H},{ }^{13} \mathrm{C}$ and ${ }^{31} \mathrm{P}$ NMR of $6\left(\mathrm{~B}^{1}=N^{3}\right.$-benzoylthymin-1-yl,

$\mathrm{B}^{2}=N^{6}$-diphenylcarbamoyl- $N^{2}$-phenylacetylguanin-9-yl) 31

${ }^{1} \mathrm{H},{ }^{13} \mathrm{C}$ and ${ }^{31} \mathrm{P}$ NMR of $27\left(\mathrm{~B}^{1}=\mathrm{B}^{2}=\right.$ thymin-1-yl)

${ }^{1} \mathrm{H},{ }^{13} \mathrm{C}$ and ${ }^{31} \mathrm{P}$ NMR of $27\left(\mathrm{~B}^{1}=\right.$ adenin-9-yl, $\mathrm{B}^{2}=$ thymin-1-yl) 33

${ }^{1} \mathrm{H},{ }^{13} \mathrm{C}$ and ${ }^{31} \mathrm{P}$ NMR of $27\left(\mathrm{~B}^{1}=\right.$ cytosin-1-yl, $\mathrm{B}^{2}=$ thymin-1-yl $)$

${ }^{1} \mathrm{H},{ }^{13} \mathrm{C}$ and ${ }^{31} \mathrm{P}$ NMR of $27\left(\mathrm{~B}^{1}=\right.$ guanin-9-yl, $\mathrm{B}^{2}=$ thymin-1-yl) 35 


\section{General Information.}

${ }^{1} \mathrm{H}$ NMR spectra were obtained at $300 \mathrm{MHz}$ with tetramethylsilane (TMS) as an internal standard in $\mathrm{CDCl}_{3}$, with TMS as an internal standard in $\mathrm{CD}_{3} \mathrm{CN}$, and with 2,2,3,3- $d_{4}$-3-(trimethylsilyl)propionic acid sodium salt $\left(\mathrm{TSP}-d_{4}\right)$ as an external standard in $\mathrm{D}_{2} \mathrm{O} .{ }^{13} \mathrm{C}$ NMR spectra were obtained at 75.45 $\mathrm{MHz}$ with $\mathrm{CDCl}_{3}$ as an internal standard $\left(\delta\right.$ 77.0) in $\mathrm{CDCl}_{3}$, with TMS as an external standard in $\mathrm{CD}_{3} \mathrm{CN}$, and with DSS as an external standard in $\mathrm{D}_{2} \mathrm{O} .{ }^{31} \mathrm{P}$ NMR spectra were obtained at $121.5 \mathrm{MHz}$ using $85 \% \mathrm{H}_{3} \mathrm{PO}_{4}$ as an external standard. Organic solvents were purified and dried by appropriate procedures. 


\section{$O^{6}$-Diphenylcarbamoyl- $N^{2}$-phenylacetyl-2'-deoxyguanosine.}

$N^{2}$-Phenylacetyl-2'-deoxyguanosine $(7.71 \mathrm{~g}, 20 \mathrm{mmol})$ was dried by repeated coevaporation with dry toluene followed by dry pyridine, and finally dissolved in dry $\mathrm{CH}_{3} \mathrm{CN}(200 \mathrm{~mL})$. To the solution was added hexamethyl disilazane $(16.8 \mathrm{~mL}, 80 \mathrm{mmol})$. After being refluxed for $3 \mathrm{~h}$, the reaction mixture was concentrated to dryness under reduced pressure, coevaporated with dry EtOH for two times, and then dissolved in dry pyridine $(200 \mathrm{~mL})$. To the solution were successively added 4dimethylaminopyridine (244 mg, $2 \mathrm{mmol}), i-\operatorname{Pr}_{2} \mathrm{NEt}(5.1 \mathrm{~mL}, 30 \mathrm{mmol})$, diphenylcarbamoyl chloride (5.56 g, $24 \mathrm{mmol})$. After being stirred at $\mathrm{rt}$ for $17 \mathrm{~h}$, the mixture was diluted with $\mathrm{MeOH}(20 \mathrm{~mL})$ and concentrated to dryness under reduced pressure. The mixture was dissolved in $\mathrm{CHCl}_{3}(200 \mathrm{~mL})$ and washed with phosphate buffer (pH 7.0, 3 x $200 \mathrm{~mL})$, and the aqueous layer was back-extracted with $\mathrm{CHCl}_{3}(3 \times 100 \mathrm{~mL})$. The organic layer and washings were combined, dried over $\mathrm{Na}_{2} \mathrm{SO}_{4}$, filtered, and concentrated to dryness under reduced pressure. To the solution of the residue was dissolved in $\mathrm{MeOH}-$ $\mathrm{CHCl}_{3}(1: 1, \mathrm{v} / \mathrm{v}, 200 \mathrm{~mL})$ was added TFA $(2 \mathrm{~mL})$, and the solution was stirred at $\mathrm{rt}$ for $10 \mathrm{~min}$. The mixture was diluted with $\mathrm{CHCl}_{3}(100 \mathrm{~mL})$ and washed with sat $\mathrm{NaHCO}_{3}$ aq $(3 \times 200 \mathrm{~mL})$, and the aqueous layer was back-extracted with $\mathrm{CHCl}_{3}(5 \times 100 \mathrm{~mL})$. The organic layer and washings were combined, dried over $\mathrm{Na}_{2} \mathrm{SO}_{4}$, filtered, and concentrated until crystallization began. After the solution was cooled at $0{ }^{\circ} \mathrm{C}$, filtration obtained $O^{6}$-diphenylcarbamoyl- $N^{2}$-phenylacetyl-2'-deoxyguanosine (10.12 g, $87 \%$ ) as a white powder: ${ }^{1} \mathrm{H}$ NMR (DMSO) $\delta 10.96(1 \mathrm{H}, \mathrm{s}), 8.65(1 \mathrm{H}, \mathrm{s}), 7.60-7.17(15 \mathrm{H}, \mathrm{m})$, $6.35(1 \mathrm{H}, \mathrm{t}, J=6.0 \mathrm{~Hz}), 5.32(1 \mathrm{H}, \mathrm{d}, J=3.0 \mathrm{~Hz}), 4.89(1 \mathrm{H}, \mathrm{t}, J=4.5 \mathrm{~Hz}), 4.43(1 \mathrm{H}, \mathrm{bs}), 3.89-3.81(1 \mathrm{H}$, bs), $3.79(2 \mathrm{H}, \mathrm{s}), 3.65-3.45(2 \mathrm{H}, \mathrm{m}), 2.81-2.68(1 \mathrm{H}, \mathrm{m}), 2.36-2.25(1 \mathrm{H}, \mathrm{m}) ;{ }^{13} \mathrm{C}$ NMR (DMSO) $\delta 151.5$, $137.5,136.9,134.5,132.6,126.8,126.3,124.1,118.0,112.9,111.9,111.8,110.8,109.8,103.0,70.7$, $70.4,66.2,65.9,53.1,53.0,44.0,25.6,22.8,22.6,22.3,22.0,21.7,21.4,21.2$; IR (KBr) 3452, 2957, $1678,1609,1563,1484,1454,1421,1399,1362,1302,1253,1220,1159,1127,1115,1086,1058$, 
1012, 951, 902, 848, 786, 731, 705, $644 \mathrm{~cm}^{-1}$. Anal. Calcd. for $\mathrm{C}_{31} \mathrm{H}_{28} \mathrm{~N}_{6} \mathrm{O}_{6} \cdot 1 / 3 \mathrm{H}_{2} \mathrm{O} \cdot 1 / 3 \mathrm{CHCl}_{3}: \mathrm{C}$, 60.08; H, 4.67; N, 13.42. Found: C, 59.85; H, 4.55; N, 13.18 .

\section{5'-Dimethoxytrityl- $O^{6}$-diphenylcarbamoyl- $N^{2}$-phenylacetyl-2'-deoxyguanosine $\left(1, B^{1}=O^{6}\right.$ -} diphenylcarbamoyl- $N^{2}$-phenylacetylguanin-9-yl).

$O^{6}$-Diphenylcarbamoyl- $N^{2}$-phenylacetyl-2'-deoxyguanosine $(8.71 \mathrm{~g}, 15 \mathrm{mmol})$ was dried by repeated coevaporation with dry pyridine and dissolved in dry pyridine $(157.5 \mathrm{~mL})$. To the solution was added $\operatorname{DMTr}-\mathrm{Cl}(5.33 \mathrm{~g}, 15.75 \mathrm{mmol})$, and the solution was stirred at $\mathrm{rt}$ for $2 \mathrm{~h}$. After concentrated to dryness under reduced pressure, the residue was dissolved in $\mathrm{CHCl}_{3}(200 \mathrm{~mL})$. The reaction mixture was washed with sat $\mathrm{NaHCO}_{3}$ aq $(3 \times 200 \mathrm{~mL})$, and the aqueous layer was back-extracted with $\mathrm{CHCl}_{3}(3 \mathrm{x}$ $200 \mathrm{~mL}$ ). The organic layer and washings were combined, dried over $\mathrm{Na}_{2} \mathrm{SO}_{4}$, filtered, and concentrated to dryness under reduced pressure. The residue was chromatographed on silica gel (150 g) using a gradient of $\mathrm{MeOH}(0-3 \%)$ in $\mathrm{CH}_{2} \mathrm{Cl}_{2}$ as an eluent. The fractions containing 5'-Dimethoxytrityl$O^{6}$-diphenylcarbamoyl- $N^{2}$-phenylacetyl-2'-deoxyguanosine $\left(\mathbf{1}, \mathrm{B}^{1}=O^{6}\right.$-diphenylcarbamoyl- $N^{2}$ phenylacetylguanin-9-yl) were combined and concentrated to dryness under reduced pressure to obtain $1\left(\mathrm{~B}^{1}=O^{6}\right.$-diphenylcarbamoyl- $N^{2}$-phenylacetylguanin-9-yl $)(11.76 \mathrm{~g}, 89 \%)$ as a colorless foam: ${ }^{1} \mathrm{H}$ $\operatorname{NMR}\left(\mathrm{CDCl}_{3}\right) \delta 8.10(1 \mathrm{H}, \mathrm{s}), 8.04(1 \mathrm{H}, \mathrm{bs}), 7.44-7.16(24 \mathrm{H}, \mathrm{m}), 6.76(4 \mathrm{H}, \mathrm{d}, J=9.0 \mathrm{~Hz}), 6.51(1 \mathrm{H}, \mathrm{t}, J$ $=6.5 \mathrm{~Hz}), 4.72-4.65(1 \mathrm{H}, \mathrm{m}), 4.18-4.12(1 \mathrm{H}, \mathrm{m}), 3.88(2 \mathrm{H}, \mathrm{s}), 3.71(6 \mathrm{H}, \mathrm{s}), 3.42-3.30(2 \mathrm{H}, \mathrm{m}), 3.07(1 \mathrm{H}$, bs), 2.73-2.62 (1H, m), 2.58-2.48 (1H, m); ${ }^{13} \mathrm{C} \mathrm{NMR}\left(\mathrm{CDCl}_{3}\right) \delta 169.6,158.5,155.9,154.4,151.5,150.4$, $144.5,142.7,141.7,135.7,135.6,133.8,130.0,130.0,129.5,129.1,128.9,128.1,127.8,127.4,126.9$, $125.7,121.7,113.1,86.5,84.4,72.3,63.9,55.2,44.4,40.6$; IR (KBr) 3407, 3062, 2933, 2837, 1742, $1620,1509,1493,1446,1410,1335,1303,1251,1185,1062,1033,1004,985,939,830,790,757,700$, $645 \mathrm{~cm}^{-1}$. Anal. Calcd. for $\mathrm{C}_{52} \mathrm{H}_{46} \mathrm{~N}_{6} \mathrm{O}_{8} \cdot \mathrm{H}_{2} \mathrm{O} \cdot 0.5 \mathrm{CHCl}_{3}: \mathrm{C}, 65.31 ; \mathrm{H}, 4.93 ; \mathrm{N}, 8.68$. Found: $\mathrm{C}, 65.33 ; \mathrm{H}$, $4.83 ; \mathrm{N}, 8.68$. 
5'-O-Dimethoxytrityl- $N^{4}$-benzoyl-2'-deoxycytidin-3'-yl dimethyl boranophosphate $\left(3, \mathrm{~B}^{1}=N^{4}\right.$ benzoylcytosin-1-yl).

${ }^{1} \mathrm{H}$ NMR $\left(\mathrm{CDCl}_{3}\right) \delta 8.66(1 \mathrm{H}, \mathrm{bs}), 8.19(1 \mathrm{H}, \mathrm{d}, J=7.5 \mathrm{~Hz}), 7.94-7.18(15 \mathrm{H}, \mathrm{m}), 6.86(4 \mathrm{H}, \mathrm{d}, J=4.2$ $\mathrm{Hz}), 6.34(1 \mathrm{H}, \mathrm{t}, J=6.5 \mathrm{~Hz}), 5.16-5.06(1 \mathrm{H}, \mathrm{m}), 4.39-4.32(1 \mathrm{H}, \mathrm{m}), 3.80(6 \mathrm{H}, \mathrm{s}), 3.72,3.68(3 \mathrm{H}, 2 \mathrm{~d}$, $J_{\mathrm{POCH}}=9.0 \mathrm{~Hz}$ and $\left.9.0 \mathrm{~Hz}\right), 3.51-3.47(2 \mathrm{H}, \mathrm{m}), 2.96-2.85(1 \mathrm{H}, \mathrm{m}), 2.42-2.30(1 \mathrm{H}, \mathrm{m}), 1-0(3 \mathrm{H}, \mathrm{bq}) ;{ }^{13} \mathrm{C}$ $\operatorname{NMR}\left(\mathrm{CDCl}_{3}\right) \delta 162.1,158.7,144.3,143.8,135.1,134.9,133.2,130.1,130.0,129.9,129.1,128.1$, $127.5,127.3,113.4,113.3,96.4,87.2,87.1,87.0,85.5,62.5,62.4,55.3,55.2,53.4,53.3,53.2,40.8 ;{ }^{31} \mathrm{P}$ NMR $\left(\mathrm{CDCl}_{3}\right) \delta$ 119.8-116.4 (m). IR (KBr) 3434, 2954, 2838, 2400, 1698, 1663, 1609, 1561, 1509, 1486, 1446, 1396, 1303, 1253, 1178, 1116, 1033, 830, 756, $705 \mathrm{~cm}^{-1}$. Anal. Calcd. for $\mathrm{C}_{39} \mathrm{H}_{43} \mathrm{BN}_{3} \mathrm{O}_{9} \mathrm{P} \cdot \mathrm{H}_{2} \mathrm{O}: \mathrm{C}, 61.83 ; \mathrm{H}, 5.99 ; \mathrm{N}, 5.55$. Found: C, 61.61; H, 5.84; N, 5.79.

5'-O-Dimethoxytrityl- $O^{6}$-diphenylcarbamoyl- $N^{2}$-phenylacetyl-2'-deoxyguanosin-3'-yl methyl 2(trimethylsilyl)ethyl boranophosphate $\left(19, \mathrm{~B}^{1}=O^{6}\right.$-diphenylcarbamoyl- $N^{2}$-phenylacetylguanin-9yl).

${ }^{1} \mathrm{H}$ NMR $\left(\mathrm{CDCl}_{3}\right) \delta 8.10(1 \mathrm{H}, \mathrm{s}), 7.91(1 \mathrm{H}, \mathrm{bs}), 7.46-7.14(24 \mathrm{H}, \mathrm{m}), 6.78(4 \mathrm{H}, \mathrm{d}, J=9.0 \mathrm{~Hz}), 6.41$ $(1 \mathrm{H}, \mathrm{t}, J=7.5 \mathrm{~Hz}), 5.21(1 \mathrm{H}, \mathrm{m}), 4.32(1 \mathrm{H}, \mathrm{m}), 4.18-4.06(2 \mathrm{H}, \mathrm{m}), 3.98(2 \mathrm{H}, \mathrm{s}), 3.74(6 \mathrm{H}, \mathrm{s}), 3.69,3.65$ $\left(3 \mathrm{H}, 2 \mathrm{~d}, J_{\mathrm{POCH}}=9.0 \mathrm{~Hz}\right.$ and $\left.9.0 \mathrm{~Hz}\right), 3.46-3.31(2 \mathrm{H}, \mathrm{m}), 2.97-2.82(1 \mathrm{H}, \mathrm{m}), 2.74-2.60(1 \mathrm{H}, \mathrm{m}), 1.11-$ $1.01(2 \mathrm{H}, \mathrm{m}), 1-0(3 \mathrm{H}, \mathrm{bq}), 0.04,0.03(9 \mathrm{H}, 2 \mathrm{~s}) ;{ }^{31} \mathrm{P} \mathrm{NMR}\left(\mathrm{CDCl}_{3}\right) \delta 119.02-115.61(\mathrm{~m})$. Anal. Calcd. for $\mathrm{C}_{58} \mathrm{H}_{64} \mathrm{BN}_{6} \mathrm{O}_{10} \mathrm{PSi}: \mathrm{C}, 64.80 ; \mathrm{H}, 6.00 ; \mathrm{N}, 7.82$. Found: C, 64.85; H, 5.91; N, 7.90.

Triethylammonium $\quad 5$ '-O-dimethoxytrityl- $N^{4}$-benzoyl-2'-deoxycytidin-3'-yl methyl boranophosphate $\left(4, B^{1}=N^{4}\right.$-benzoylcytosin-1-yl).

${ }^{1} \mathrm{H}$ NMR $\left(\mathrm{CDCl}_{3}\right) \delta 12.50(1 \mathrm{H}, \mathrm{bs}), 8.70(1 \mathrm{H}, \mathrm{bs}), 8.24(1 \mathrm{H}, \mathrm{q}, J=7.0 \mathrm{~Hz}), 7.90,7.87(2 \mathrm{H}, 2 \mathrm{~m}), 7.65-$ $7.11(13 \mathrm{H}, \mathrm{m}), 6.94-6.79(4 \mathrm{H}, \mathrm{m}), 6.36-6.26(1 \mathrm{H}, \mathrm{m}), 5.04(1 \mathrm{H}, \mathrm{m}), 4.41-4.31(1 \mathrm{H}, \mathrm{m}), 3.79,3.78(6 \mathrm{H}$, 
2s), 3.48, $3.44\left(3 \mathrm{H}, 2 \mathrm{~d}, J_{\mathrm{POCH}}=12.0 \mathrm{~Hz}\right.$ and $\left.12.0 \mathrm{~Hz}\right), 3.52-3.44(2 \mathrm{H}, \mathrm{m}), 3.04(6 \mathrm{H}, \mathrm{q}, J=7.0 \mathrm{~Hz}), 2.90-$ $2.78(1 \mathrm{H}, \mathrm{m}), 2.41-2.28(1 \mathrm{H}, \mathrm{m}), 1.31(9 \mathrm{H}, \mathrm{t}, J=7.5 \mathrm{~Hz}), 1-0(3 \mathrm{H}, \mathrm{bq}) ;{ }^{13} \mathrm{C} \mathrm{NMR}\left(\mathrm{CDCl}_{3}\right) \delta 161.9$ $158.6,144.9,144.1,144.0,135.5,135.4,135.3,135.2,133.0,130.1,129.0,128.2,128.0,127.5,127.1$, $126.6,113.3,113.2,109.7,109.5,96.2,87.4,87.2,86.9,86.8,86.1,85.7,72.9,72.7,72.1,71.9,62.8$, 55.2, 55.1, 50.3, 50.1, 45.4, 45.3, 41.4, 8.6, 8.5, 8.4; ${ }^{31} \mathrm{P}$ NMR $\left(\mathrm{CDCl}_{3}\right) \delta$ 98.93-93.67 (m). IR (KBr) $3424,2942,2838,2679,2363,1698,1655,1608,1562,1486,1395,1303,1253,1177,1116,1071$, 1033, 915, 831, 790, 757, 706, $660 \mathrm{~cm}^{-1}$. Anal. Calcd. for $\mathrm{C}_{44} \mathrm{H}_{56} \mathrm{BN}_{4} \mathrm{O}_{9} \mathrm{P} \cdot 2.5 \mathrm{H}_{2} \mathrm{O}: \mathrm{C}, 60.62 ; \mathrm{H}, 7.05 ; \mathrm{N}$, 6.43. Found: C, 60.85; H, 6.79; N, 6.47.

Tributylammonium

deoxyguanosin-3'-yl methyl boranophosphate $\left(20, \mathbf{B}^{1}=O^{6}\right.$-diphenylcarbamoyl- $N^{2}$ phenylacetylguanin-9-yl).

${ }^{1} \mathrm{H}$ NMR $\left(\mathrm{CDCl}_{3}\right) \delta 12.52(1 \mathrm{H}, \mathrm{bs}), 8.08(1 \mathrm{H}, \mathrm{bs}), 8.02(1 \mathrm{H}, \mathrm{d}, J=9.0 \mathrm{~Hz}), 7.51-7.09(24 \mathrm{H}, \mathrm{m}), 6.75$

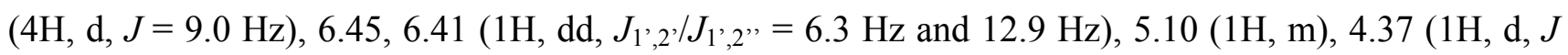
$=15.0 \mathrm{~Hz}), 4.02(2 \mathrm{H}, \mathrm{s}), 3.69(6 \mathrm{H}, \mathrm{s}), 3.49,3.42\left(3 \mathrm{H}, 2 \mathrm{~d}, J_{\mathrm{POCH}}=12.0 \mathrm{~Hz}\right.$ and $\left.9.0 \mathrm{~Hz}\right), 3.38-3.28(2 \mathrm{H}$, m), 2.93-2.80 (6H, m), 2.82-2.63 (2H, m), 1.71-1.52 (6H, m), $1.33(6 \mathrm{H}, \mathrm{q}, J=8.0 \mathrm{~Hz}), 0.92(9 \mathrm{H}, \mathrm{t}, J=$ 7.5 Hz), 1-0 (3H, bq); ${ }^{13} \mathrm{C} \mathrm{NMR}\left(\mathrm{CDCl}_{3}\right) \delta 170.0,158.3,155.8,154.5,151.6,150.3,144.4,142.4,142.3$, $141.6,135.6,135.5,134.2,130.1,129.9,129.4,129.3,129.1,129.0,128.7,128.6,128.0,127.7,127.0$, $126.7,126.0,125.5,121.2,121.1,113.5,113.0,86.4,86.3,86.2,86.1,85.7,85.6,84.4,84.2,73.9,73.6$, $63.8,63.7,55.0,51.5,50.0,49.9,49.8,49.7,43.7,40.0,39.8,24.9,20.0,13.5 ;{ }^{31} \mathrm{P} \mathrm{NMR}\left(\mathrm{CDCl}_{3}\right) \delta$ 99.09-93.23 (m). IR (KBr) 3442, 2961, 2874, 2838, 2363, 1743, 1619, 1509, 1494, 1455, 1384, 1335, 1303, 1251, 1223, 1185, 1065, 1033, 987, 943, 831, 789, 758, 701, $645 \mathrm{~cm}^{-1}$. Anal. Calcd. for $\mathrm{C}_{65} \mathrm{H}_{79} \mathrm{BN}_{7} \mathrm{O}_{10} \mathrm{P}: \mathrm{C}, 67.29 ; \mathrm{H}, 6.86 ; \mathrm{N}, 8.45$. Found: C, 67.00; H, 6.98; N, 8.22. 
5'-O-Dimethoxytrityl- $N^{4}$-benzoyl-2'-deoxycytidin-3'-yl 3'- $O, N^{3}$-dibenzoylthymidin-5'-yl methyl boranophosphate $\left(6, \mathrm{~B}^{1}=N^{4}\right.$-benzoylcytosin-1-yl, $\mathrm{B}^{2}=N^{3}$-benzoylthymin-1-yl).

${ }^{1} \mathrm{H}$ NMR $\left(\mathrm{CDCl}_{3}\right) \delta 8.69(1 \mathrm{H}, \mathrm{bs}), 8.20-8.13(1 \mathrm{H}, \mathrm{m}), 8.06-7.16(26 \mathrm{H}, \mathrm{m}), 6.91-6.82(4 \mathrm{H}, \mathrm{m}), 6.54-$ $6.46(1 \mathrm{H}, \mathrm{m}), 6.34(1 \mathrm{H}, \mathrm{t}, J=6.0 \mathrm{~Hz}), 5.55-5.40(1 \mathrm{H}, \mathrm{m}), 5.23-5.14(1 \mathrm{H}, \mathrm{m}), 4.52-4.24(4 \mathrm{H}, \mathrm{m}), 3.93-$ $3.64(9 \mathrm{H}, \mathrm{m}), 3.56-3.46(2 \mathrm{H}, \mathrm{m}), 3.06-2.89(1 \mathrm{H}, \mathrm{m}), 2.69-2.52(1 \mathrm{H}, \mathrm{m}), 2.47-2.25(1 \mathrm{H}, \mathrm{m}), 2.03,2.00$ (3H, 2s), 1-0 (3H, bq) $;{ }^{13} \mathrm{C} \mathrm{NMR}\left(\mathrm{CDCl}_{3}\right) \delta 168.7,166.0,162.6,162.2,158.7,149.4144 .3,143.8,130.5$, $130.0,129.7,129.1,128.9,128.8,128.6,128.4,128.0,127.5,127.2,113.4,112.1,96.4,87.3,87.0,85.7$, $85.4,84.7,84.6,82.8,78.1,77.8,74.7,65.9,65.4,64.8,62.7,62.5,55.2,54.2,54.0,40.9,40.7,37.3$, 37.2, 29.7, 14.0, 12.6; ${ }^{31} \mathrm{P}$ NMR $\left(\mathrm{CDCl}_{3}\right) \delta$ 121.21-117.55 (m). IR (KBr) 3441, 2953, 2837, 2401, 1751, $1703,1663,1561,1509,1485,1448,1397,1300,1254,1178,1110,1071,1031,828,763,714 \mathrm{~cm}^{-1}$. Anal. Calcd. for $\mathrm{C}_{62} \mathrm{H}_{61} \mathrm{BN}_{5} \mathrm{O}_{15} \mathrm{P} \cdot 2 / 3 \mathrm{CHCl}_{3}$ : C, 60.82; H, 5.02; N, 5.66. Found: $\mathrm{C}, 60.42 ; \mathrm{H}, 5.15 ; \mathrm{N}$, 5.59 .

5'-O-Dimethoxytrityl- $O^{6}$-diphenylcarbamoyl- $N^{2}$-phenylacetyl-2'-deoxyguanosin-3'-yl $\quad 3$ ' $-O, N^{3}-$ dibenzoylthymidin-5'-yl methyl boranophosphate $\left(6, \mathrm{~B}^{1}=O^{6}\right.$-diphenylcarbamoyl- $N^{2}$ phenylacetylguanin-9-yl, $B^{2}=N^{3}$-benzoylthymin-1-yl).

${ }^{1} \mathrm{H}$ NMR $\left(\mathrm{CDCl}_{3}\right) \delta$ 8.13-7.11 (36H, m), 6.82-6.71 (4H, m), 6.55-6.36 (2H, m), 5.54-5.42 (1H, m), 5.38-5.28 (1H, m), 4.50-4.20 (4H, m), 3.89-3.64 (11H, m), 3.52-3.34 (2H, m), 3.08-2.88 (1H, m), 2.79$2.67(1 \mathrm{H}, \mathrm{m}), 2.67-2.50(1 \mathrm{H}, \mathrm{m}), 2.43-2.21(1 \mathrm{H}, \mathrm{m}), 2.03,2.01(3 \mathrm{H}, 2 \mathrm{~s}), 1-0(3 \mathrm{H}, \mathrm{bq}) ;{ }^{13} \mathrm{C}$ NMR $\left(\mathrm{CDCl}_{3}\right) \delta 168.7,166.0,162.6,158.6,156.0,154.3,151.8,150.3,149.4,144.2,143.9,142.4,142.2$ $141.7,135.3,135.1,134.7,134.6,134.0,133.8,132.1,131.4,130.4,130.0,129.6,129.5,129.4,129.1$, $128.8,128.6,128.0,127.9,127.3,127.0,125.6,121.6,113.2,112.1,112.0,86.8,85.2,84.9,84.7,84.5$, $84.3,83.0,78.5,78.3,74.8,66.0,65.6,63.2,55.1,54.3,54.2,44.2,39.0,37.3,37.1,12.6 ;{ }^{31} \mathrm{P}$ NMR $\left(\mathrm{CDCl}_{3}\right) \delta$ 120.94-117.46 (m). IR (KBr) 3440, 2952, 2837, 2401, 1750, 1705, 1664, 1622, 1509, 1493, 
1450, 1396, 1272, 1179, 1109, 1032, 831, 790, 761, $700 \mathrm{~cm}^{-1}$. Anal. Calcd. for $\mathrm{C}_{77} \mathrm{H}_{72} \mathrm{BN}_{8} \mathrm{O}_{16} \mathrm{P} \cdot 1 / 3 \mathrm{CHCl}_{3}: \mathrm{C}, 64.19 ; \mathrm{H}, 5.04 ; \mathrm{N}, 7.74$. Found: $\mathrm{C}, 64.26 ; \mathrm{H}, 5.23 ; \mathrm{N}, 7.64$.

5'-O-Dimethoxytrityl- $N^{3}$-benzoylthymidin-3'-yl $\quad 3$ '- $O, N^{3}$-dibenzoylthymidin-5'-yl methyl boranophosphate $\left(6, B^{1}=B^{2}=N^{3}\right.$-benzoylthymin-1-yl).

${ }^{1} \mathrm{H}$ NMR $\left(\mathrm{CDCl}_{3}\right)$ $\delta$ 8.00-7.26 (26H, m), 6.90-6.83 (4H, m), 6.49-6.41 (2H, m), 5.51-5.35 (1H, m), 5.30-5.23 (1H, m), 4.47-4.19 (4H, m), 3.49, $3.72\left(3 \mathrm{H}, 2 \mathrm{~d}, J_{\mathrm{POCH}}=12.0 \mathrm{~Hz}\right.$ and $\left.12.0 \mathrm{~Hz}\right), 3.80-3.77(6 \mathrm{H}$, m), 3.55-3.46 (2H, m), 2.73-2.21 (4H, m), 2.00, $1.99(3 \mathrm{H}, 2 \mathrm{~s}), 1.51,1.50(3 \mathrm{H}, 2 \mathrm{~s}), 1-0(3 \mathrm{H}, \mathrm{bq}) ;{ }^{13} \mathrm{C}$ $\operatorname{NMR}\left(\mathrm{CDCl}_{3}\right) \delta 168.8,168.7,166.0,162.6,162.5,158.8,149.3,149.2,143.9,135.0,134.9,134.6$, $134.5,133.7,131.4,130.8,130.4,130.0,129.7,129.1,128.7,128.5,128.1,128.0,127.3,113.4,112.1$, $112.0,111.7,111.6,87.4,84.9,84.8,84.7,84.6,84.5,82.9,82.8,82.7,78.8,78.7,78.6,78.5,74.5,65.8$, 65.5, 63.3, 63.2, 55.2, 54.1, 39.7, 39.5, 37.2, 37.0, 12.5, 11.8; ${ }^{31} \mathrm{P} \mathrm{NMR}\left(\mathrm{CDCl}_{3}\right) \delta 121.18-117.40(\mathrm{~m})$. IR (KBr) 3448, 2954, 2837, 2401, 1751, 1705, 1664, 1601, 1509, 1447, 1396, 1366, 1254, 1178, 1108 , 1071, 1032, 914, 829, 791, 763, 715, $687 \mathrm{~cm}^{-1}$. Anal. Calcd. for $\mathrm{C}_{63} \mathrm{H}_{62} \mathrm{BN}_{4} \mathrm{O}_{16} \mathrm{P} \cdot 2 \mathrm{CHCl}_{3}: \mathrm{C}, 61.28 ; \mathrm{H}$, 5.38; N, 4.40. Found: C, 61.02; H, 5.10; N, 4.45.

5'-O-Dimethoxytrityl- $N^{3}$-benzoylthymidin-3'-yl 3'-O-t-butyldimethylsilyl- $N^{4}$-benzoylcytidin-5'yl methyl boranophosphate $\left(6, B^{1}=N^{3}\right.$-benzoylthymin-1-yl, $B^{2}=N^{4}$-benzoylcytosin-1-yl).

${ }^{1} \mathrm{H}$ NMR $\left(\mathrm{CDCl}_{3}\right) \delta$ 9.00-8.65 (1H, bs), 8.09-7.22 (23H, m), 6.91-6.82 (4H, m), 6.49-6.41 (1H, m), 6.26-6.19 $(1 \mathrm{H}, \mathrm{m}), 5.31-5.20(1 \mathrm{H}, \mathrm{m}), 4.39-4.00(5 \mathrm{H}, \mathrm{m}), 3.79(6 \mathrm{H}, \mathrm{s}), 3.75,3.67\left(3 \mathrm{H}, 2 \mathrm{~d}, J_{\mathrm{POCH}}=11.1\right.$ $\mathrm{Hz}$ and $11.4 \mathrm{~Hz}), 3.62-3.46(2 \mathrm{H}, \mathrm{m}), 2.71-2.45(3 \mathrm{H}, \mathrm{m}), 2.25-2.21(1 \mathrm{H}, \mathrm{m}), 1.50,1.47(3 \mathrm{H}, 2 \mathrm{~s}), 0.88$, $0.86(9 \mathrm{H}, 2 \mathrm{~s}), 0.10-0.04(6 \mathrm{H}, \mathrm{m}), 1-0(3 \mathrm{H}, \mathrm{bq}) ;{ }^{13} \mathrm{C} \mathrm{NMR}\left(\mathrm{CDCl}_{3}\right) \delta 168.8,162.6,162.2,158.7,154.5$, $149.2,143.9,134.9,133.0,131.4,130.8,130.3,129.9,129.4,129.0,128.8,128.7,128.2,128.0,127.9$, $127.5,127.2,113.3,111.6,111.5,96.6,87.3,86.9,85.1,84.9,84.8,84.4,78.5,78.3,70.2,68.0,64.7$, $63.1,55.2,53.7,41.4,39.5,39.4,38.6,30.2,29.5,28.8,25.5,23.6,22.8,17.8,13.9,11.7,10.8,-4.8,-$ 
5.1; ${ }^{31} \mathrm{P}$ NMR $\left(\mathrm{CDCl}_{3}\right) \delta$ 121.86-117.78 (m). Anal. Calcd. for $\mathrm{C}_{61} \mathrm{H}_{71} \mathrm{BN}_{5} \mathrm{O}_{14} \mathrm{PSi} \cdot 2 / 3 \mathrm{CHCl}_{3}: \mathrm{C}, 59.36 ; \mathrm{H}$, 5.79; N, 5.61. Found: C, 59.53; H, 5.89; N, 5.55.

5'-O-Dimethoxytrityl- $N^{3}$-benzoylthymidin-3'-yl 3'-O-benzoyl- $N^{6}$-diphenylcarbamoyl- $N^{2}-$ phenylacetyl-2'-deoxyguanosin-5'-yl methyl boranophosphate $\left(6, B^{1}=N^{3}\right.$-benzoylthymin-1-yl, $B^{2}$ $=N^{6}$-diphenylcarbamoyl- $N^{2}$-phenylacetylguanin-9-yl).

${ }^{1} \mathrm{H}$ NMR $\left(\mathrm{CDCl}_{3}\right) \delta$ 8.20-7.18 (37H, m), 6.89-6.80 (4H, m), 6.48-6.35 (2H, m), 5.82-5.69 (1H, m), 5.26-5.16 (1H, m), 4.45-4.37 (2H, m), 4.28, $4.20(2 \mathrm{H}, 2 \mathrm{~s}), 3.92(2 \mathrm{H}, \mathrm{s}), 3.79-3.74(6 \mathrm{H}, \mathrm{m}), 3.48-3.38$ $(2 \mathrm{H}, \mathrm{m}), 3.30-3.13(1 \mathrm{H}, \mathrm{m}), 2.76-2.35(3 \mathrm{H}, \mathrm{m}), 1.48-1.43(3 \mathrm{H}, \mathrm{m}), 1-0(3 \mathrm{H}, \mathrm{bq}),{ }^{13} \mathrm{C} \mathrm{NMR}\left(\mathrm{CDCl}_{3}\right) \delta$ $169.0,165.9,165.8,162.8,158.8,156.1,154.3,151.7,150.3,149.3,144.8,144.0,142.9,142.8,141.6$, $135.2,135.0,133.8,133.6,131.5,130.5,130.0,129.7,129.5,129.1,129.0,128.5,128.1,128.0,127.4$, $127.2,127.0,121.8,113.4,111.6,87.3,85.0,84.9,84.7,84.6,83.7,83.6,78.2,74.9,66.1,63.3,63.2$, 55.3, 53.7, 44.5, 44.5, 39.6, 39.4, 36.7, 11.8, 0.0; ${ }^{31} \mathrm{P}$ NMR $\left(\mathrm{CDCl}_{3}\right) \delta 120.50-117.06(\mathrm{~m})$. Anal. Calcd. for $\mathrm{C}_{77} \mathrm{H}_{72} \mathrm{BN}_{8} \mathrm{O}_{16} \mathrm{P} \cdot 1 / 2 \mathrm{CHCl}_{3}: \mathrm{C}, 63.45 ; \mathrm{H}, 4.98 ; \mathrm{N}, 7.64$. Found: $\mathrm{C}, 63.71 ; \mathrm{H}, 5.25 ; \mathrm{N}, 7.62$.

Triethylammonium 2'-deoxyadenosin-3'-yl thymidin-5'-yl boranophosphate (27, B $^{1}=$ adenin-9yl, $B^{2}=$ thymin-1-yl).

${ }^{1} \mathrm{H}$ NMR $\left(\mathrm{D}_{2} \mathrm{O}\right) \delta 8.22(1 \mathrm{H}, \mathrm{s}), 8.08(1 \mathrm{H}, \mathrm{s}), 7.55,7.45(1 \mathrm{H}, 2 \mathrm{~s}), 6.33(1 \mathrm{H}, \mathrm{t}, J=6.0 \mathrm{~Hz}), 6.20,6.15$ $\left(1 \mathrm{H}, \mathrm{dd}, J_{1^{\prime}, 2^{\prime}} / J_{1^{\prime}, 2^{\prime}}=6.8 \mathrm{~Hz}\right.$ and $\left.13.4 \mathrm{~Hz}\right), 5.03-4.93(1 \mathrm{H}, \mathrm{m}), 4.56-4.48(1 \mathrm{H}, \mathrm{m}), 4.33-4.24(1 \mathrm{H}, \mathrm{m})$, 4.15-4.04 (2H, m), 3.90-3.75 (2H, m), 3.58, 3.55 (1H, 2s), $3.18(6 \mathrm{H}, \mathrm{q}, J=7.0 \mathrm{~Hz}), 2.88-2.60(2 \mathrm{H}, \mathrm{m})$, 2.32-2.23 (2H, m), $1.71(3 \mathrm{H}, \mathrm{s}), 1.26(9 \mathrm{H}, \mathrm{t}, J=7.5 \mathrm{~Hz}), 1-0(3 \mathrm{H}, \mathrm{bq}) ;{ }^{13} \mathrm{C} \mathrm{NMR}\left(\mathrm{D}_{2} \mathrm{O}\right) \delta 168.6,168.5$, $157.8,154.5,154.0,150.9,150.6,143.3,142.9,139.6,139.4,121.6,113.9,113.8,89.8,89.7,89.6,89.4$, $89.3,89.2,89.1,87.9,87.8,87.7,87.6,87.5,87.1,76.4,76.1,75.0,72.9,65.5,64.3,63.9,63.8,57.8$, $52.8,52.8,49.4,42.6,41.6,41.5,41.3,33.0,28.3,17.5,14.4,14.2,13.4,11.4,10.9 ;{ }^{31} \mathrm{P}$ NMR $\left(\mathrm{D}_{2} \mathrm{O}\right)$ 
$\delta$ 95.88-90.73 (m). $\mathrm{FAB}^{+} \mathrm{m} / z$ calcd for $\mathrm{C}_{20} \mathrm{H}_{30} \mathrm{BN}_{7} \mathrm{O}_{9} \mathrm{P}[\mathrm{M}+\mathrm{H}]^{+}$554.1936, found 554.1923. UV $\left(\mathrm{H}_{2} \mathrm{O}\right)$ $\lambda_{\max }: 261.2 \mathrm{~nm} ; \varepsilon_{261.2}: 21800$.

Triethylammonium 2'-deoxycytidin-3'-yl thymidin-5' -yl boranophosphate $\left(27, \mathbf{B}^{1}=\right.$ cytosine-1yl, $B^{2}=$ thymin-1-yl).

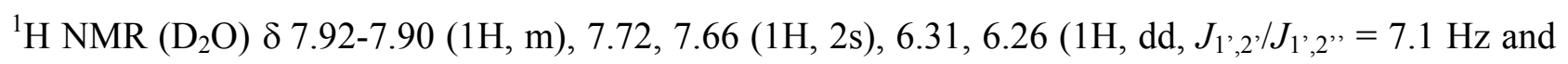
$14.3 \mathrm{~Hz}), 6.18(1 \mathrm{H}, \mathrm{t}, J=7.5 \mathrm{~Hz}), 6.07,6.04(1 \mathrm{H}, 2 \mathrm{~m}), 4.90-4.82(1 \mathrm{H}, \mathrm{m}), 4.58-4.51(1 \mathrm{H}, \mathrm{m}), 4.22-4.00$ (4H, m), 3.88-3.71 (2H, m), $3.19(6 \mathrm{H}, \mathrm{q}, J=7.0 \mathrm{~Hz}), 2.61-2.28(4 \mathrm{H}, \mathrm{m}), 1.90(3 \mathrm{H}, \mathrm{s}), 1.27(9 \mathrm{H}, \mathrm{t}, J=$ $7.5 \mathrm{~Hz}), 1-0(3 \mathrm{H}, \mathrm{bq}) ;{ }^{13} \mathrm{C} \mathrm{NMR}\left(\mathrm{D}_{2} \mathrm{O}\right) \delta 169.1,169.0,166.4,156.9,154.3,145.1,140.0,139.9,114.2$, 114.1, 98.4, 89.1, 88.8, 88.2, 88.1, 87.8, 87.6, 75.3, 74.6, 73.5, 73.4, 65.5, 64.4, 64.3, 63.5, 49.4, 41.7, 41.5, 41.4, 14.6, 14.5, 11.0; ${ }^{31} \mathrm{P}$ NMR $\left(\mathrm{D}_{2} \mathrm{O}\right) \delta 95.56-90.36(\mathrm{~m}) . \mathrm{FAB}^{+} \mathrm{m} / z$ calcd for $\mathrm{C}_{19} \mathrm{H}_{30} \mathrm{BN}_{5} \mathrm{O}_{10} \mathrm{P}$ $[\mathrm{M}+\mathrm{H}]^{+}$530.1823, found 530.1837. UV $\left(\mathrm{H}_{2} \mathrm{O}\right) \lambda_{\max }: 269.6 \mathrm{~nm} ; \varepsilon_{269.6}: 15700$. 
Supporting Info Page 13
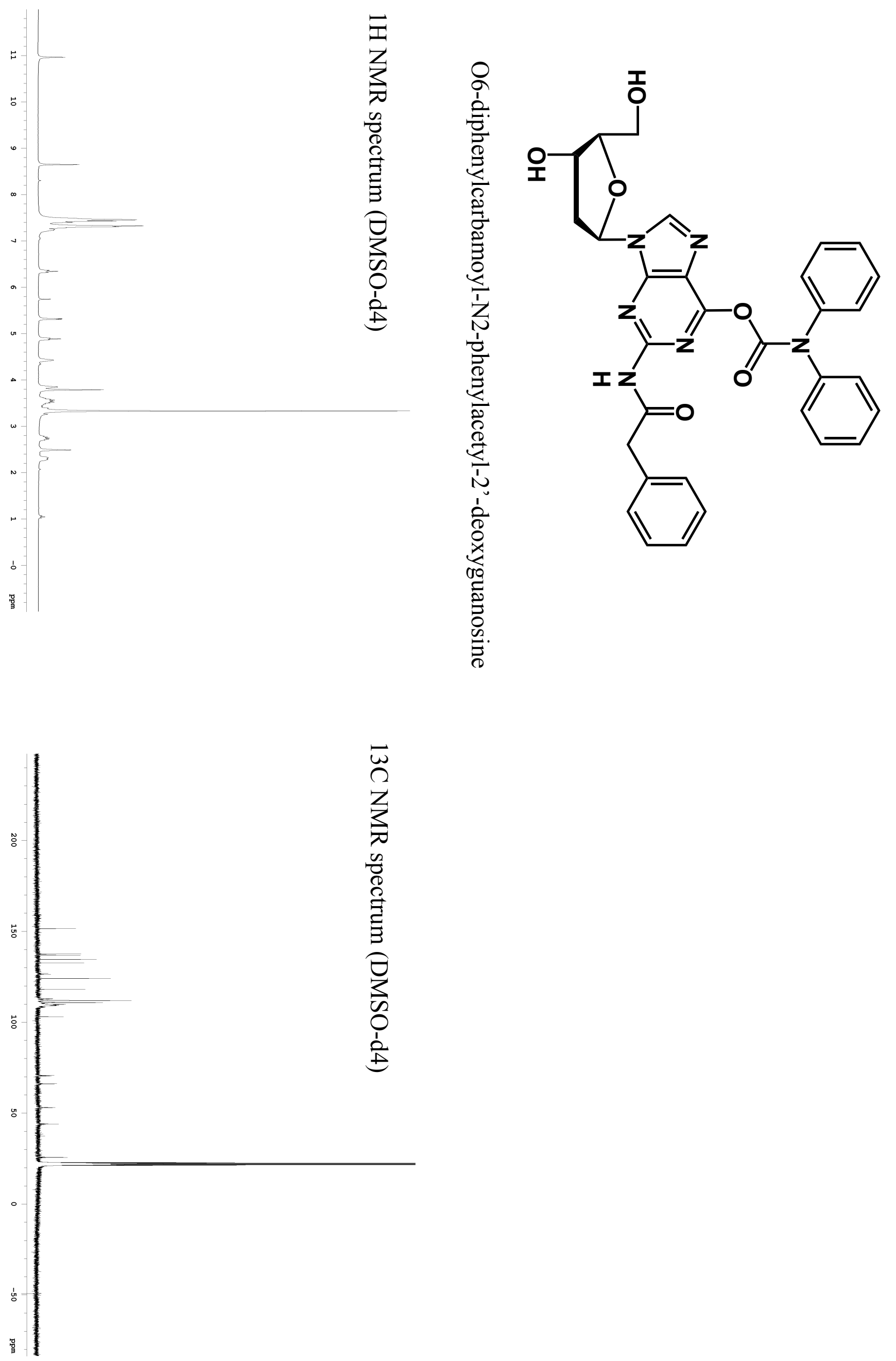
Supporting Info Page 14
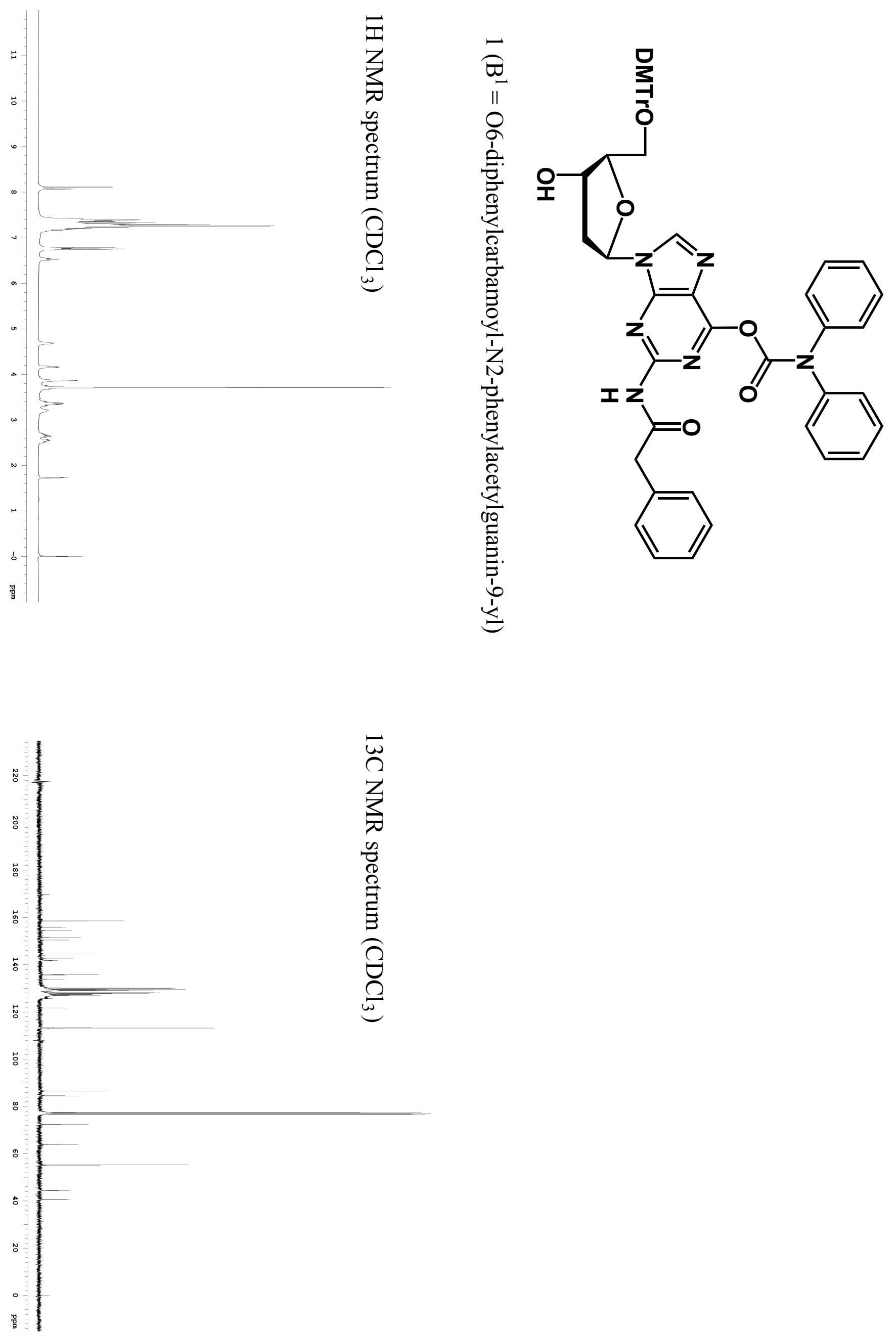
Supporting Info Page 15
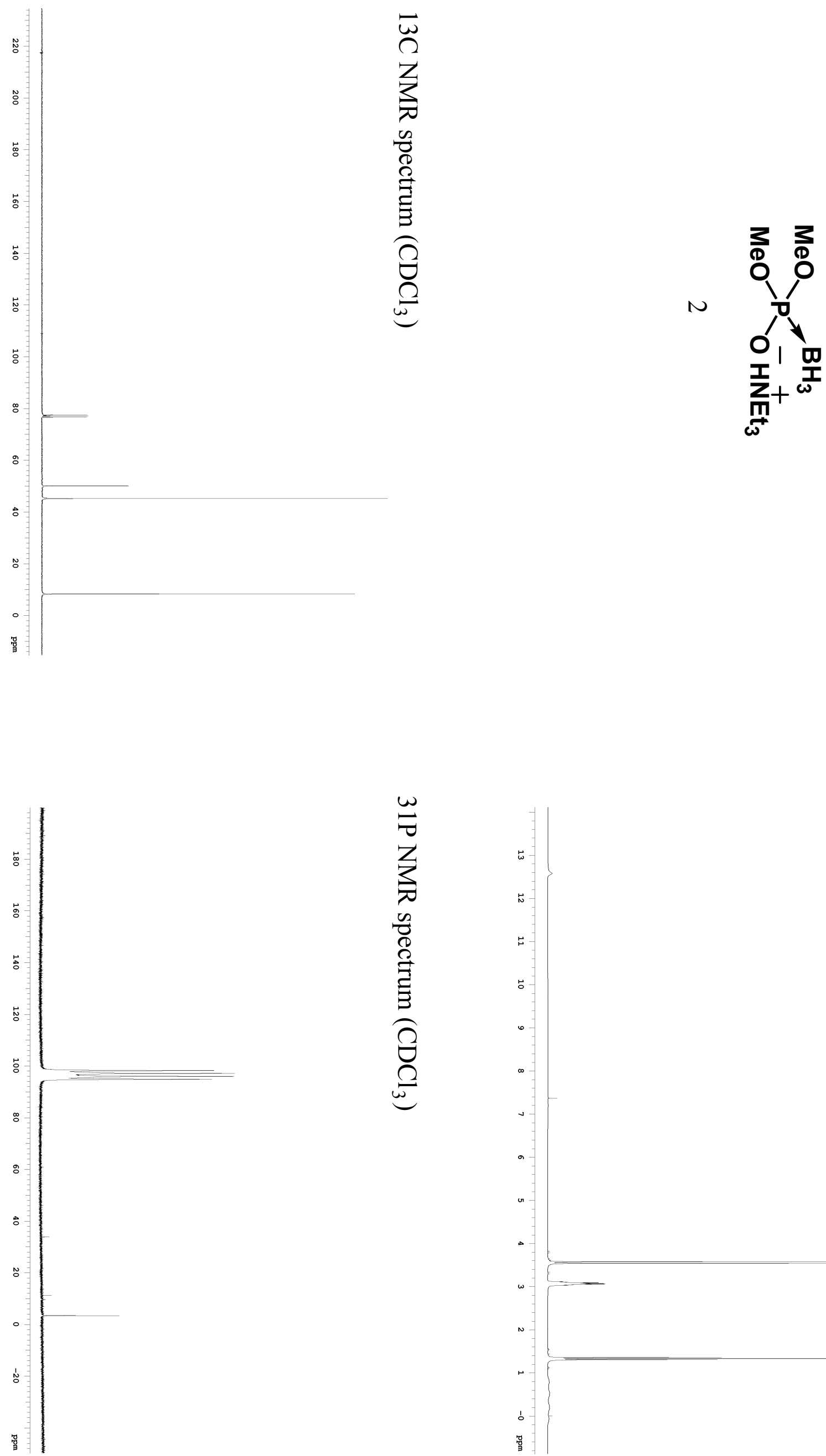

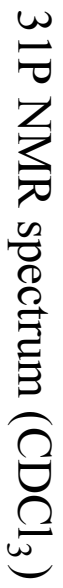

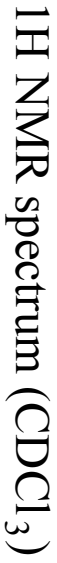


Supporting Info Page 16
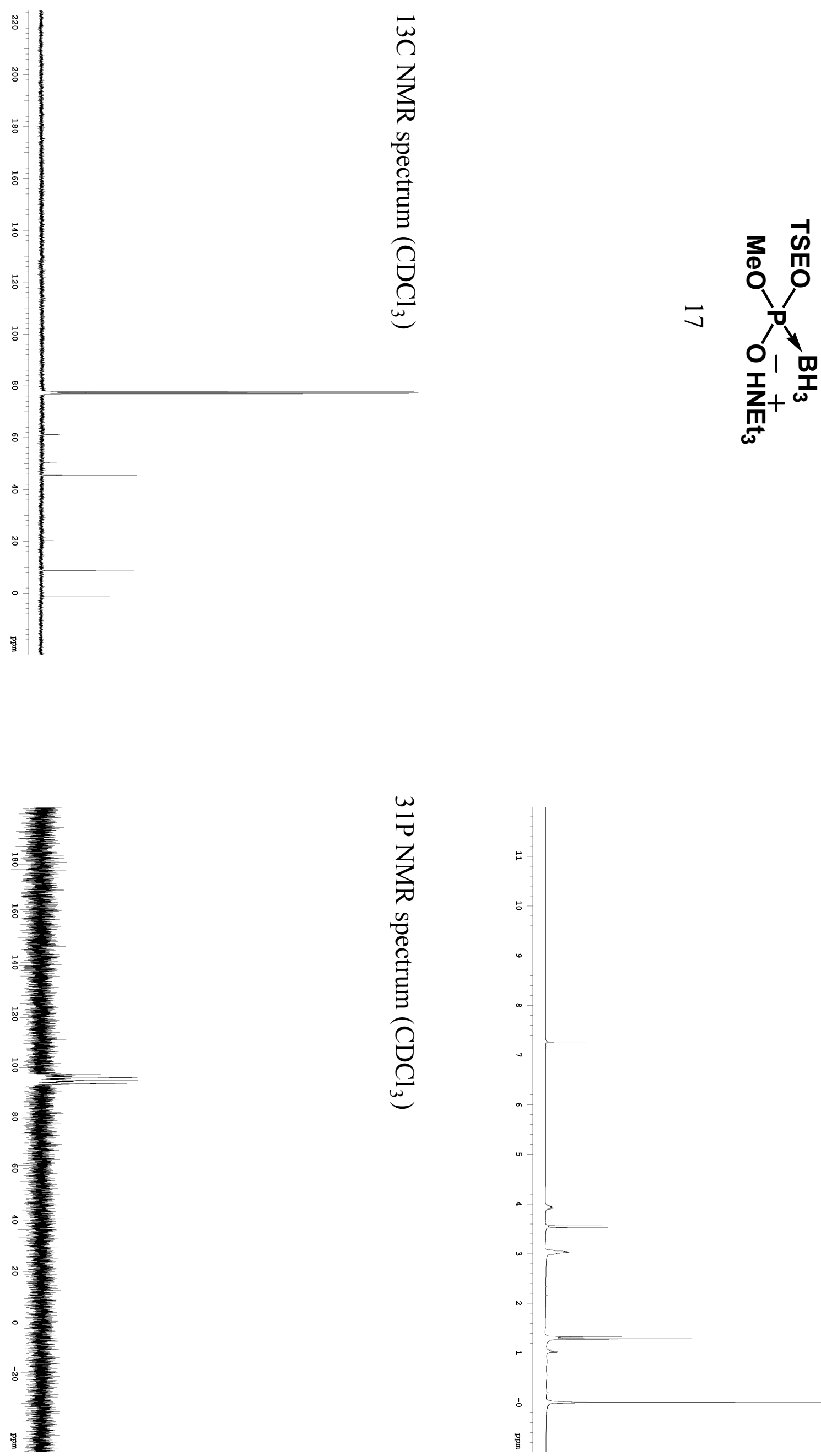

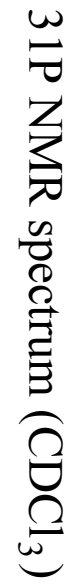

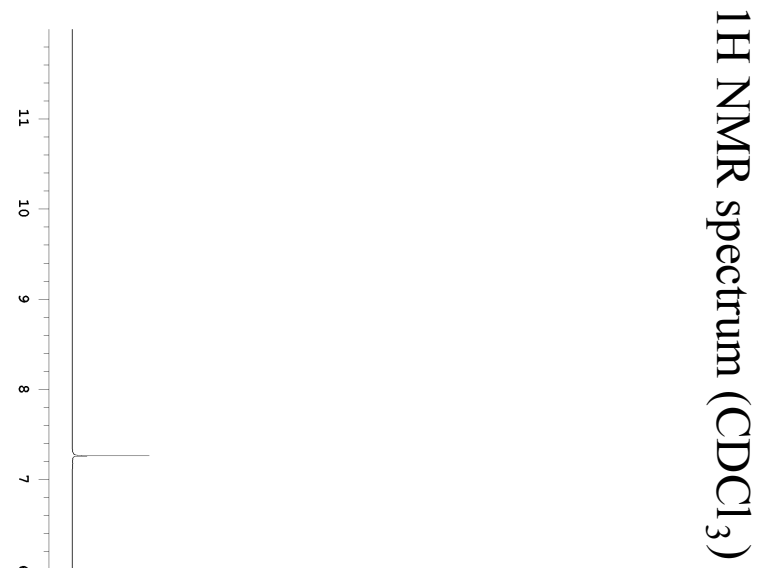


Supporting Info Page 17
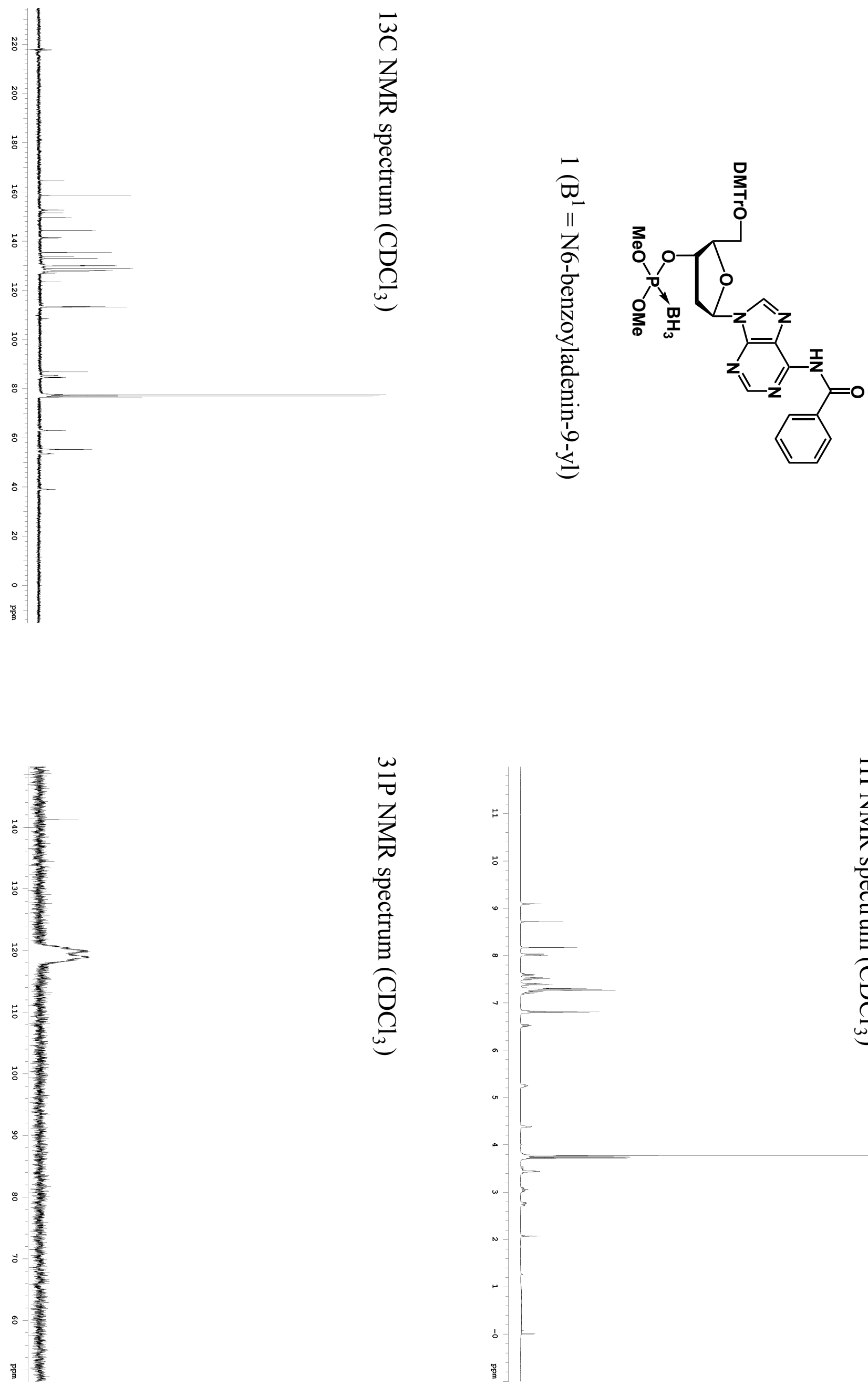

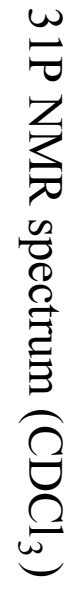

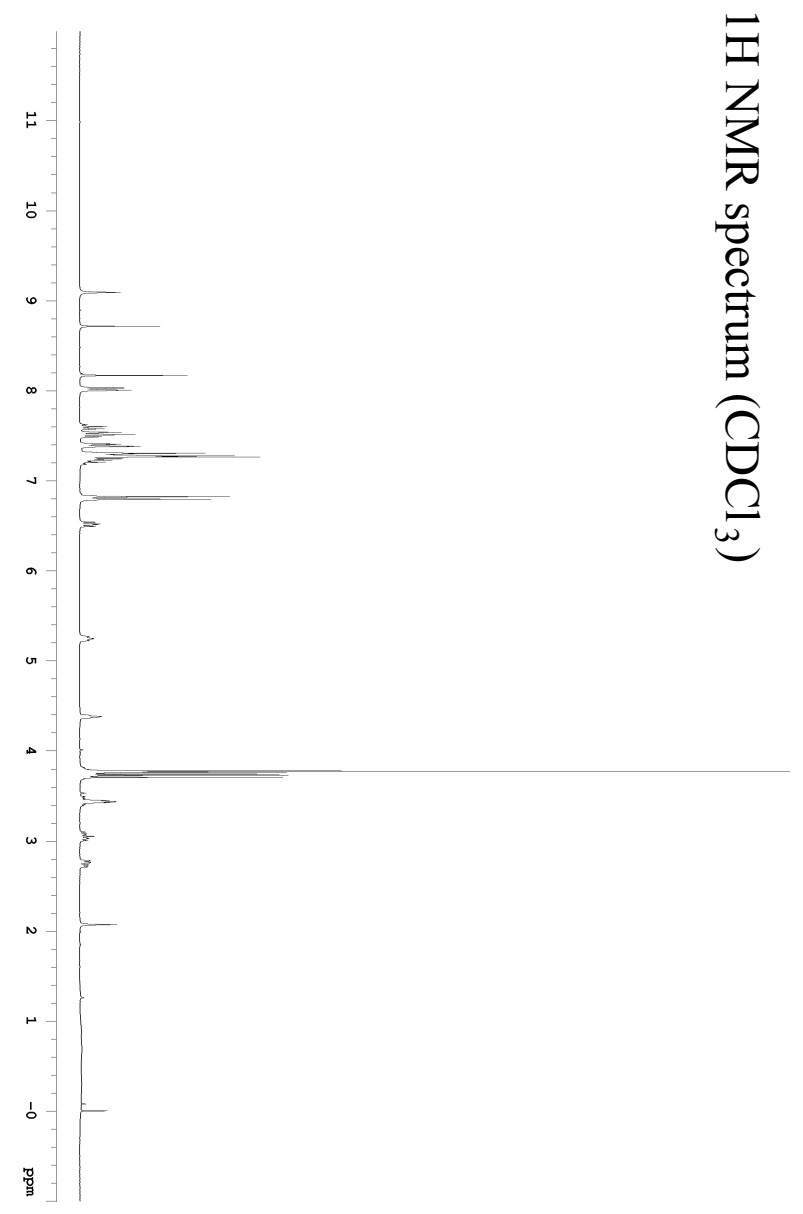


Supporting Info Page 18
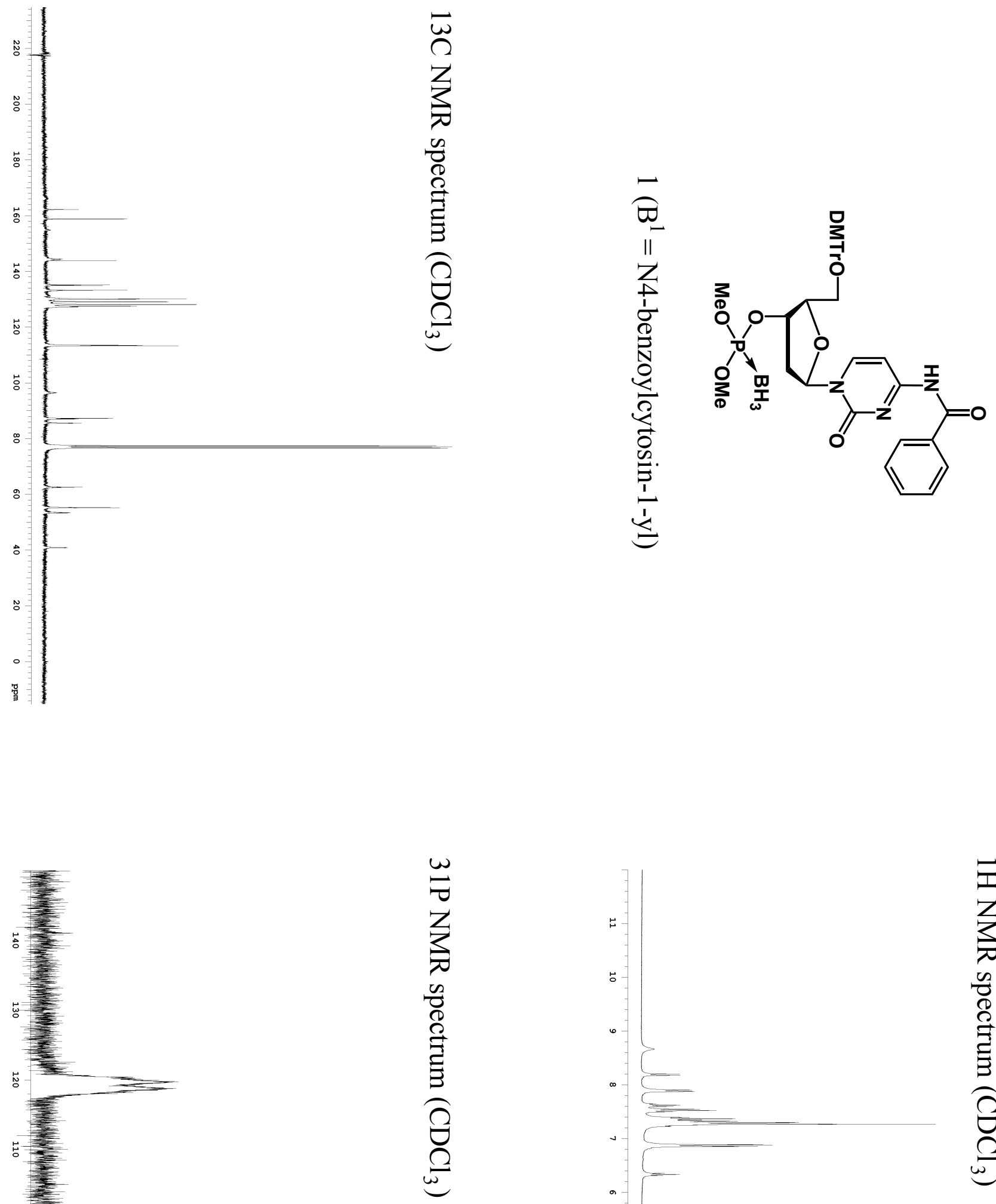

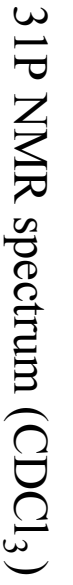

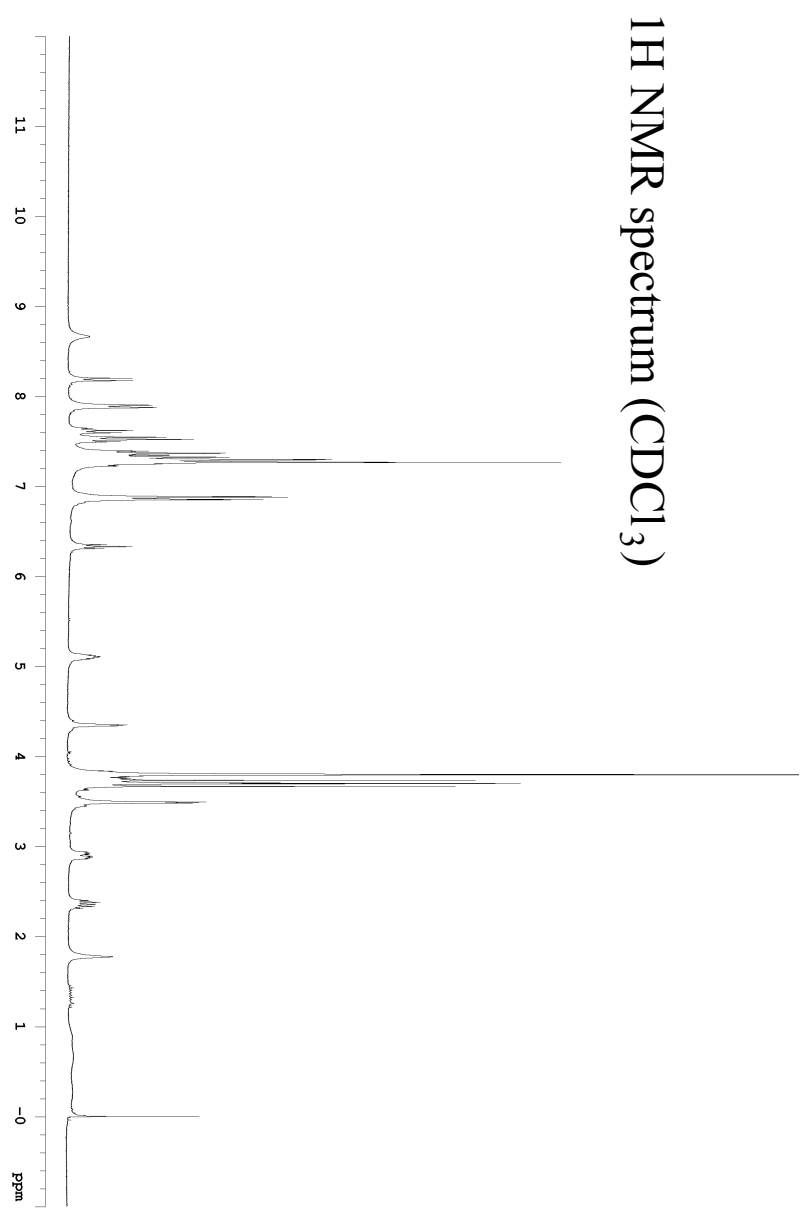


Suppoting Info Page 19
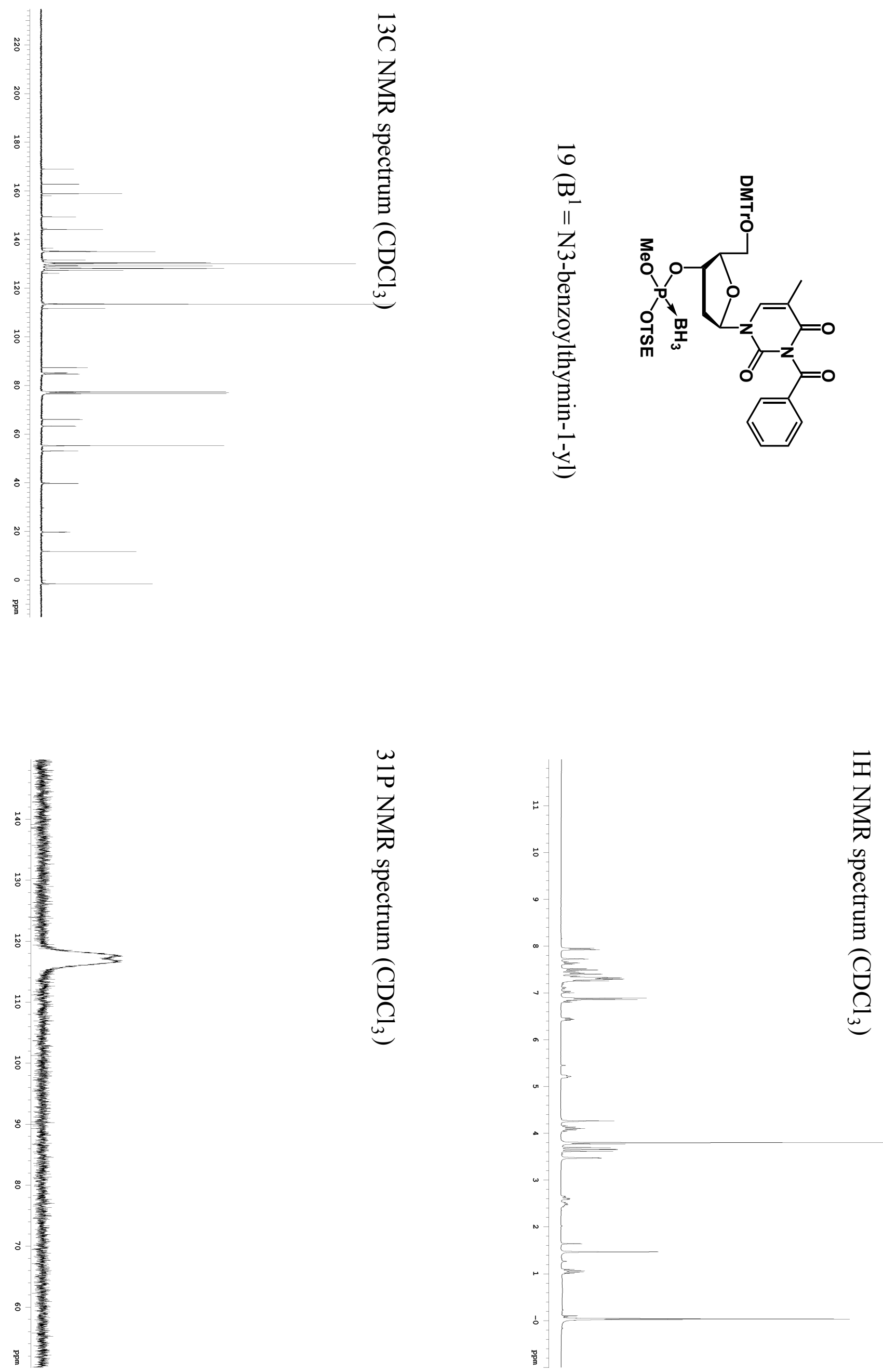


$$
11^{1}
$$


Supporting Info Page 21
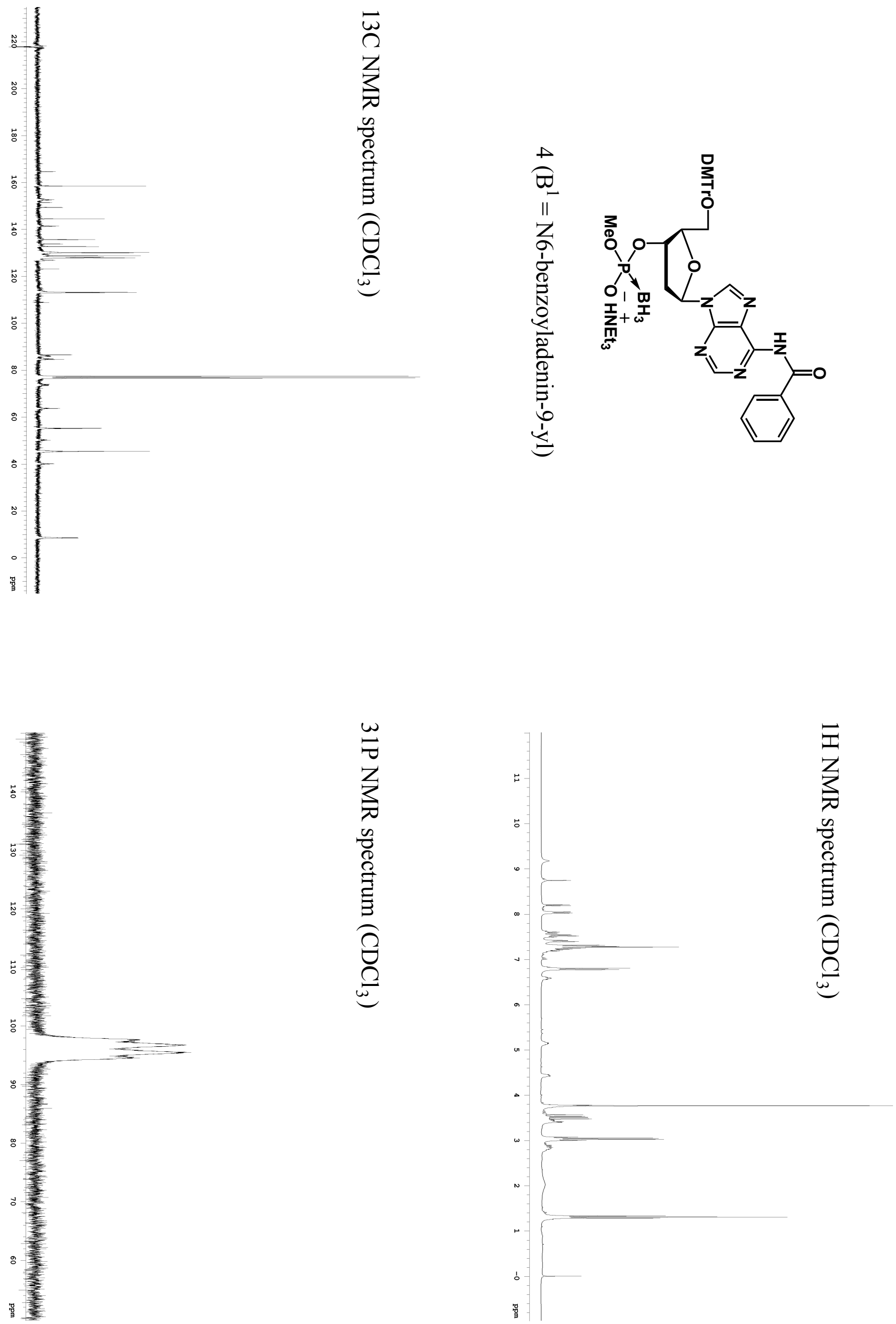
Supporting Info Page 22

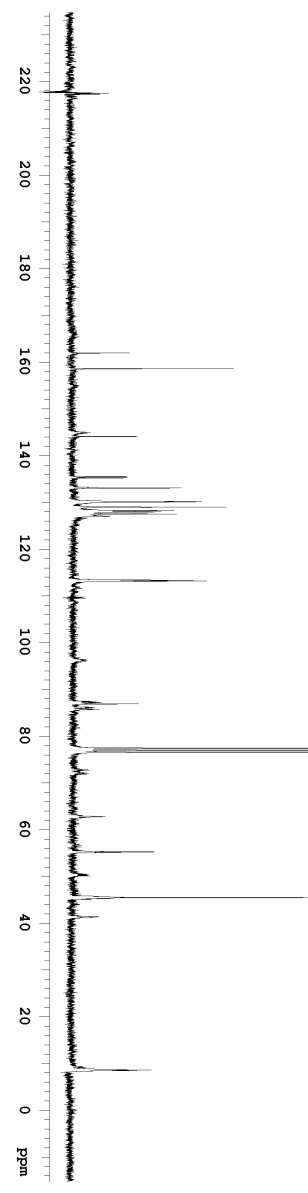

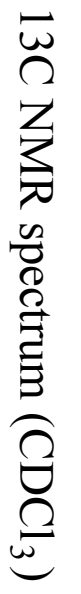

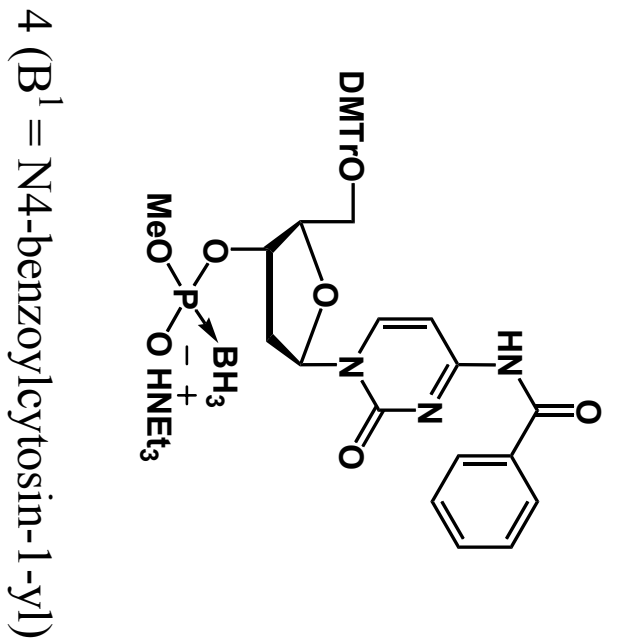

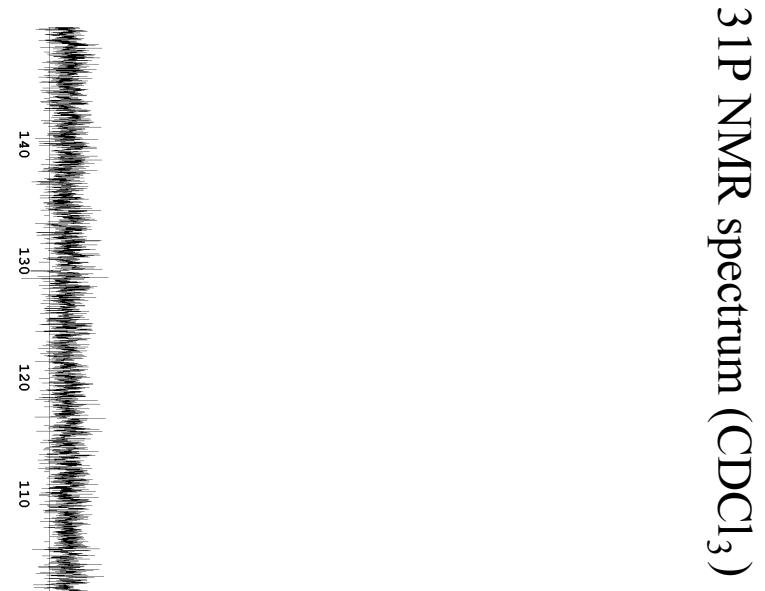
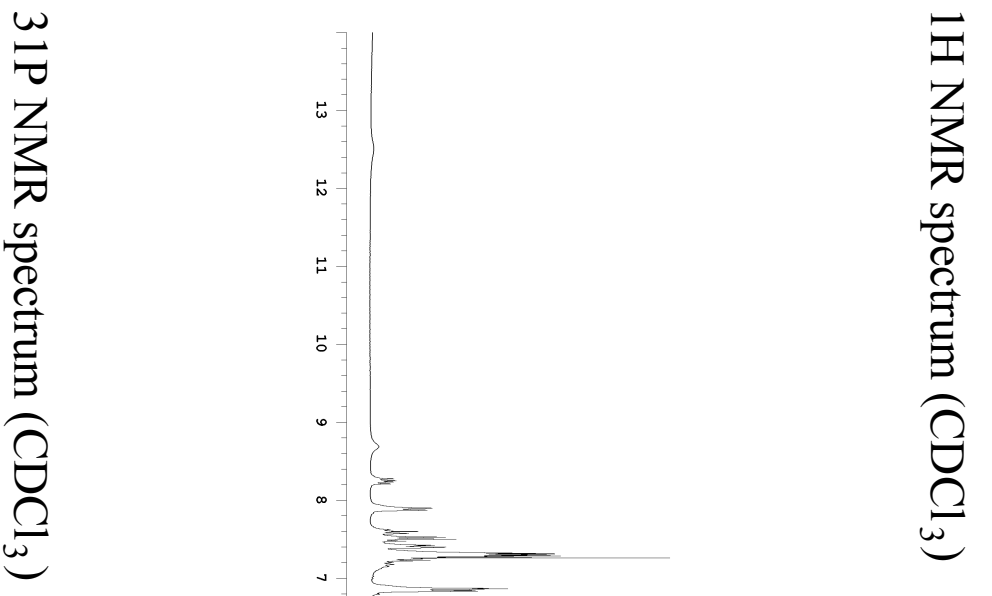
Supporting Info Page 23
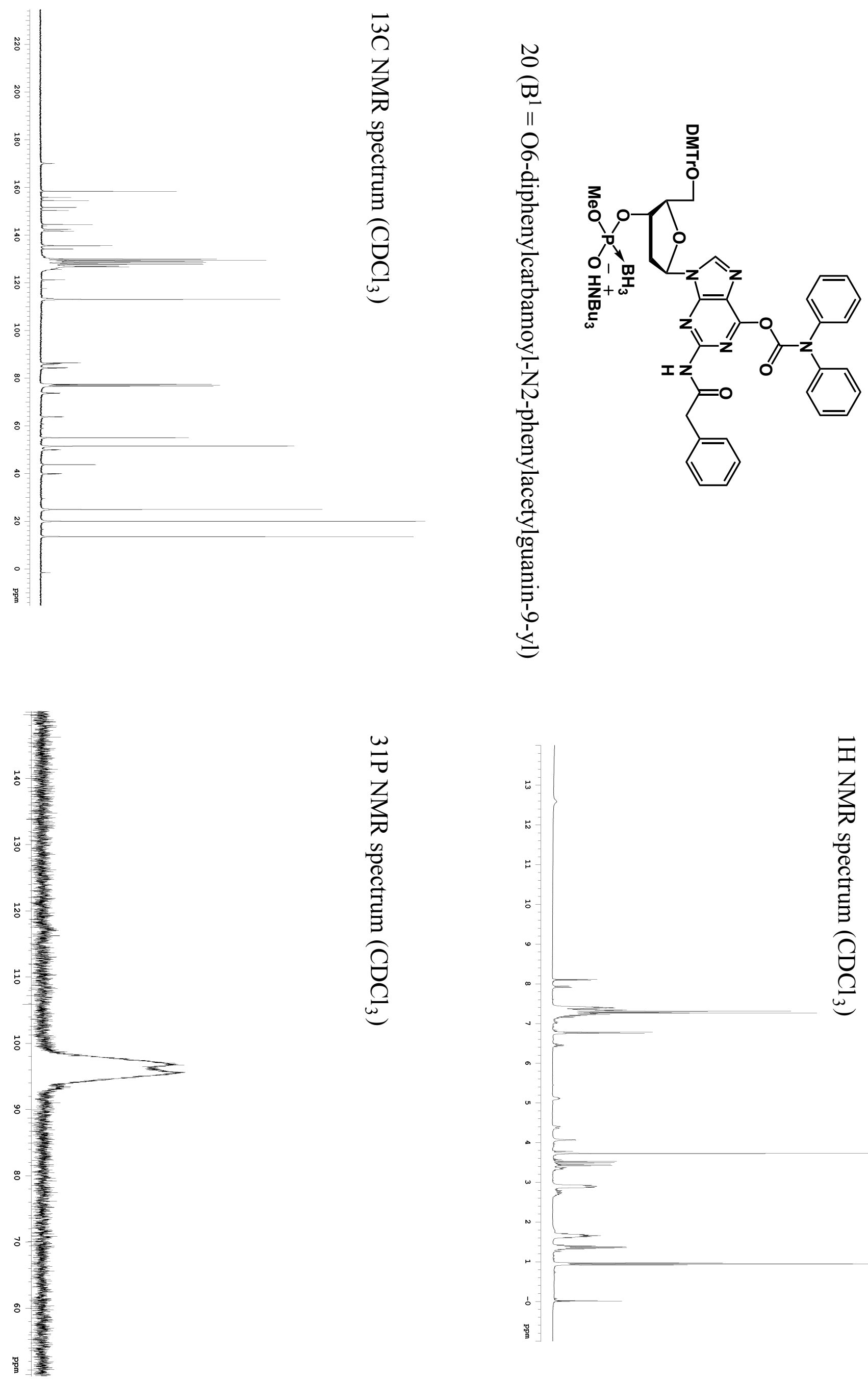

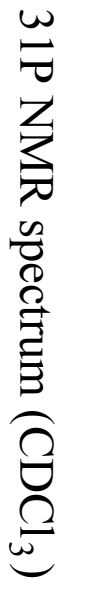

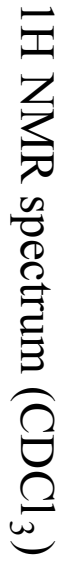


Supporting Info Page 24
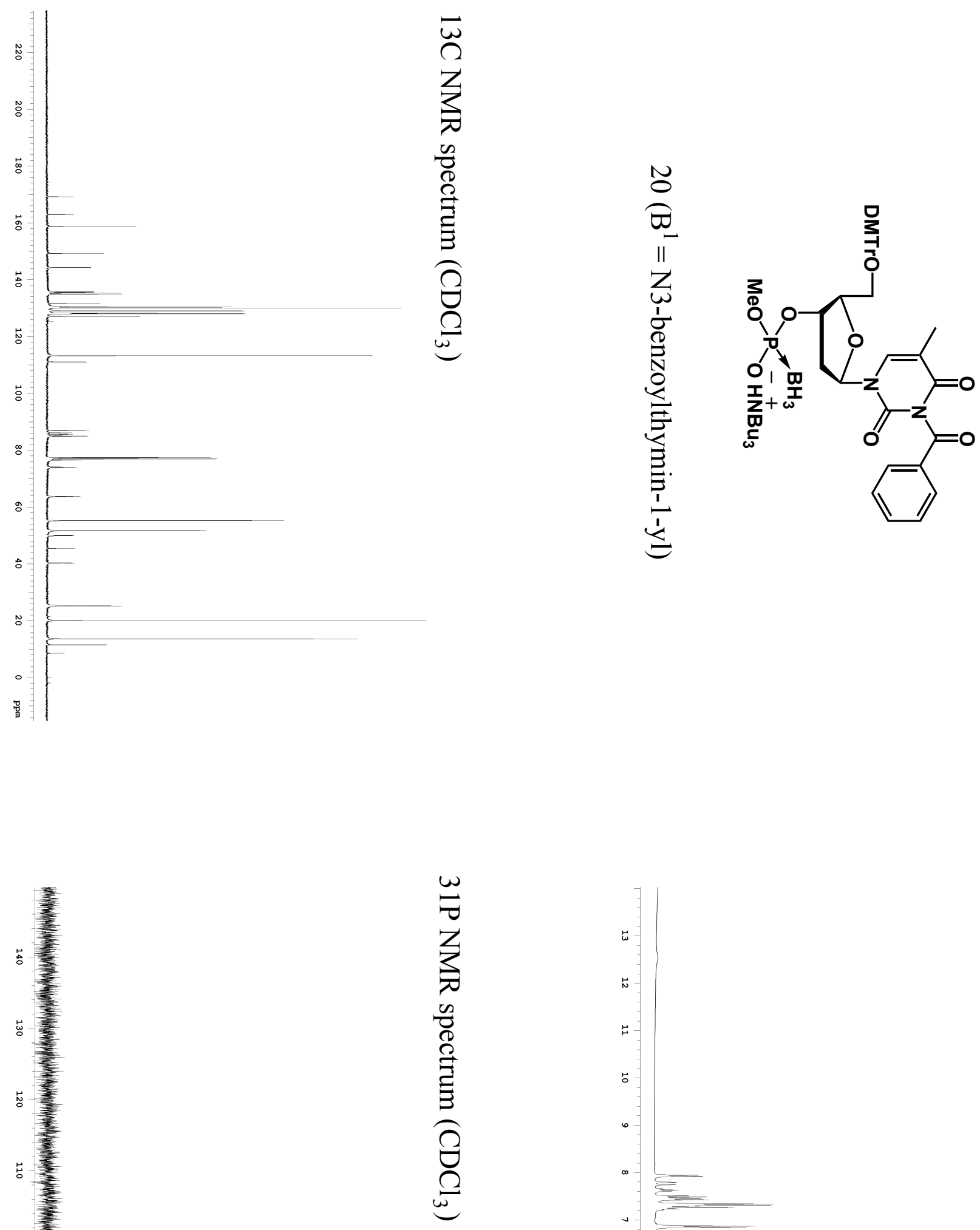

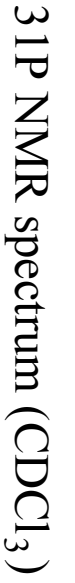
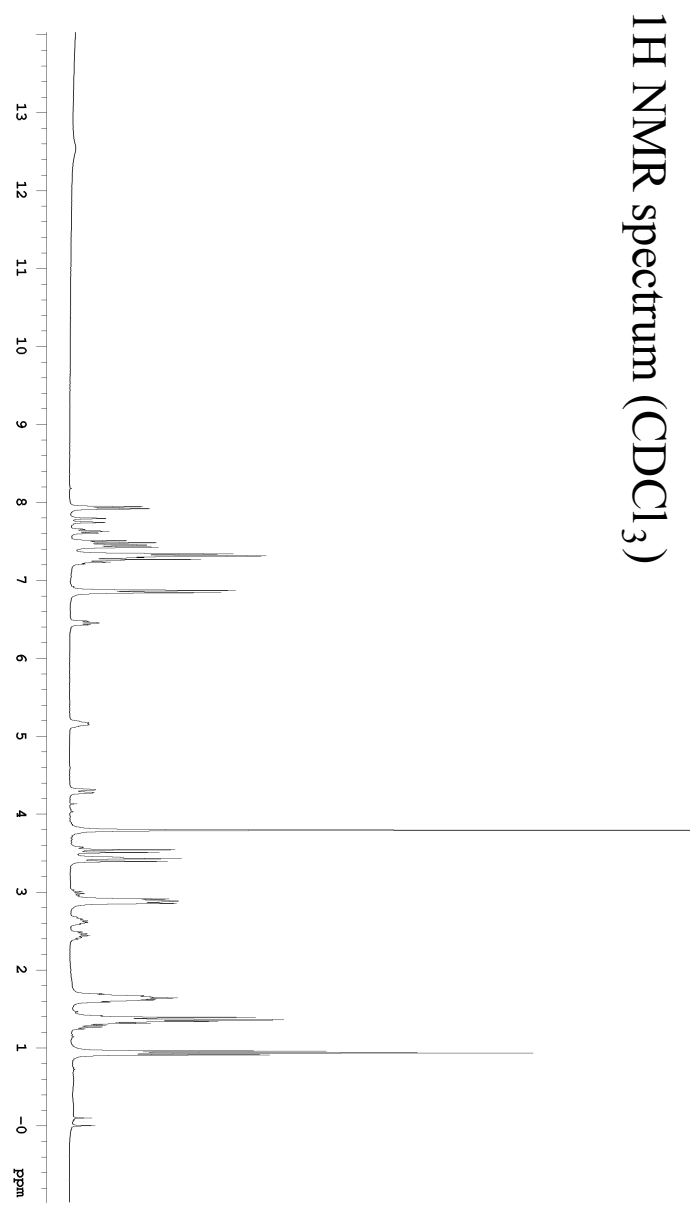
Supporting Info Page 25

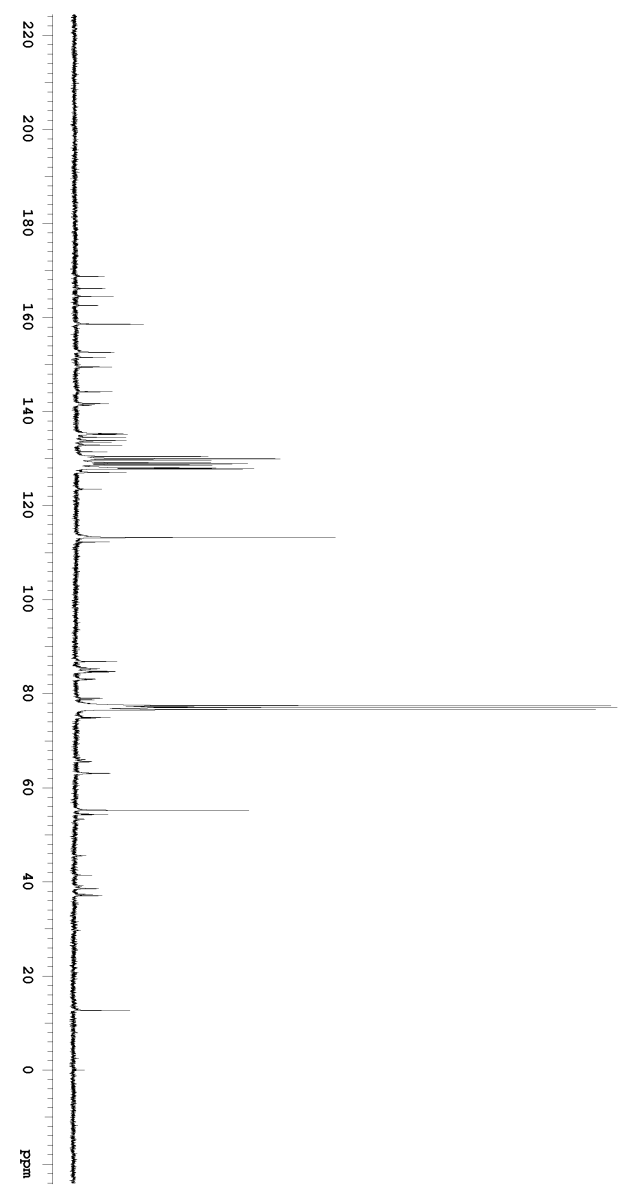

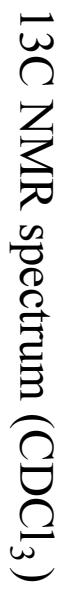

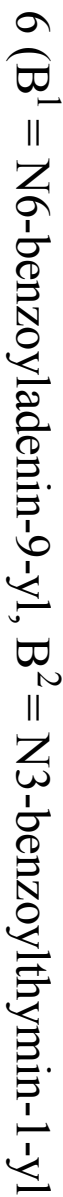

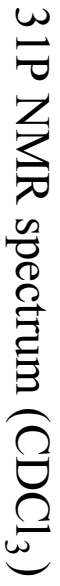
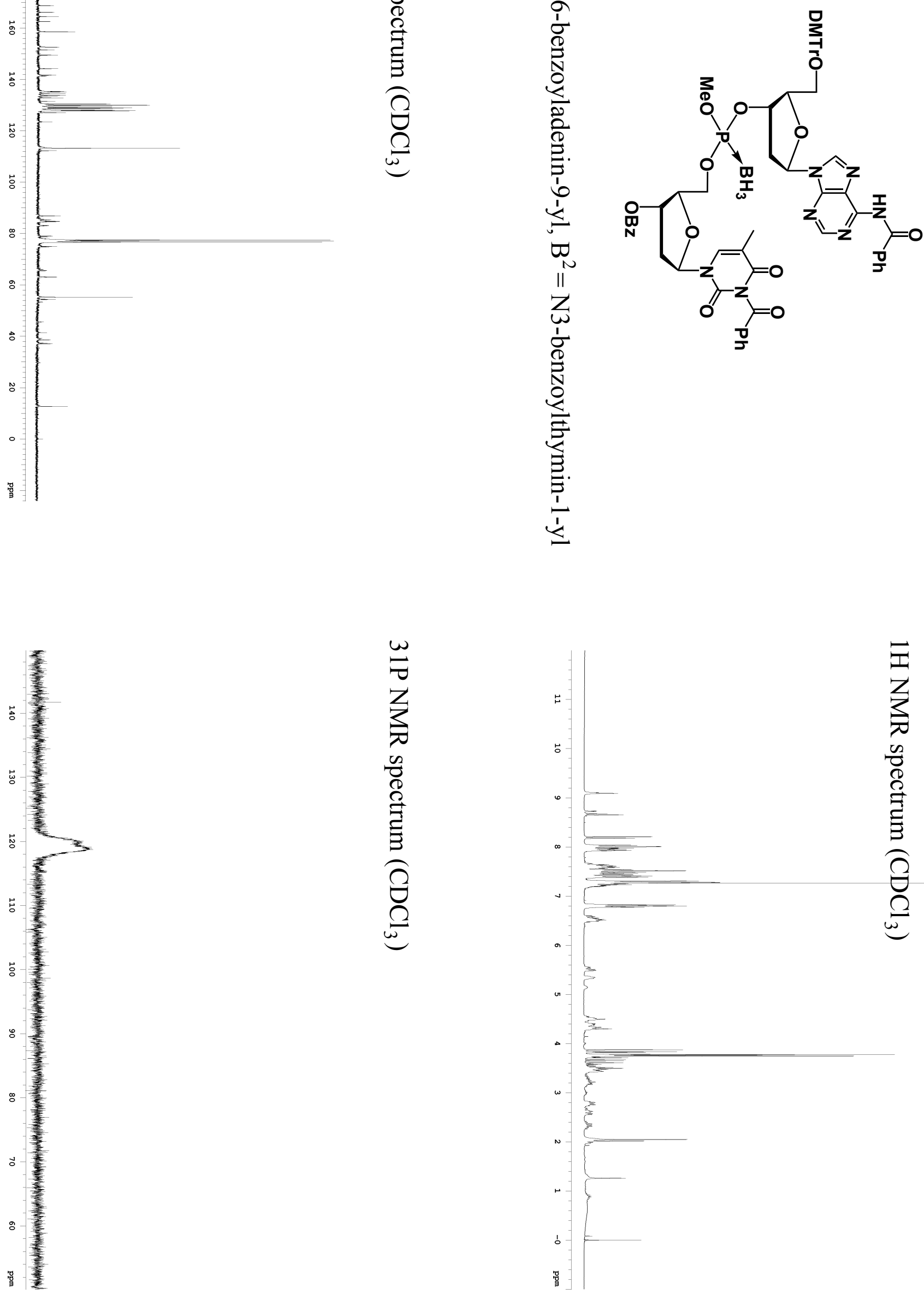
Supporting Info Page 26
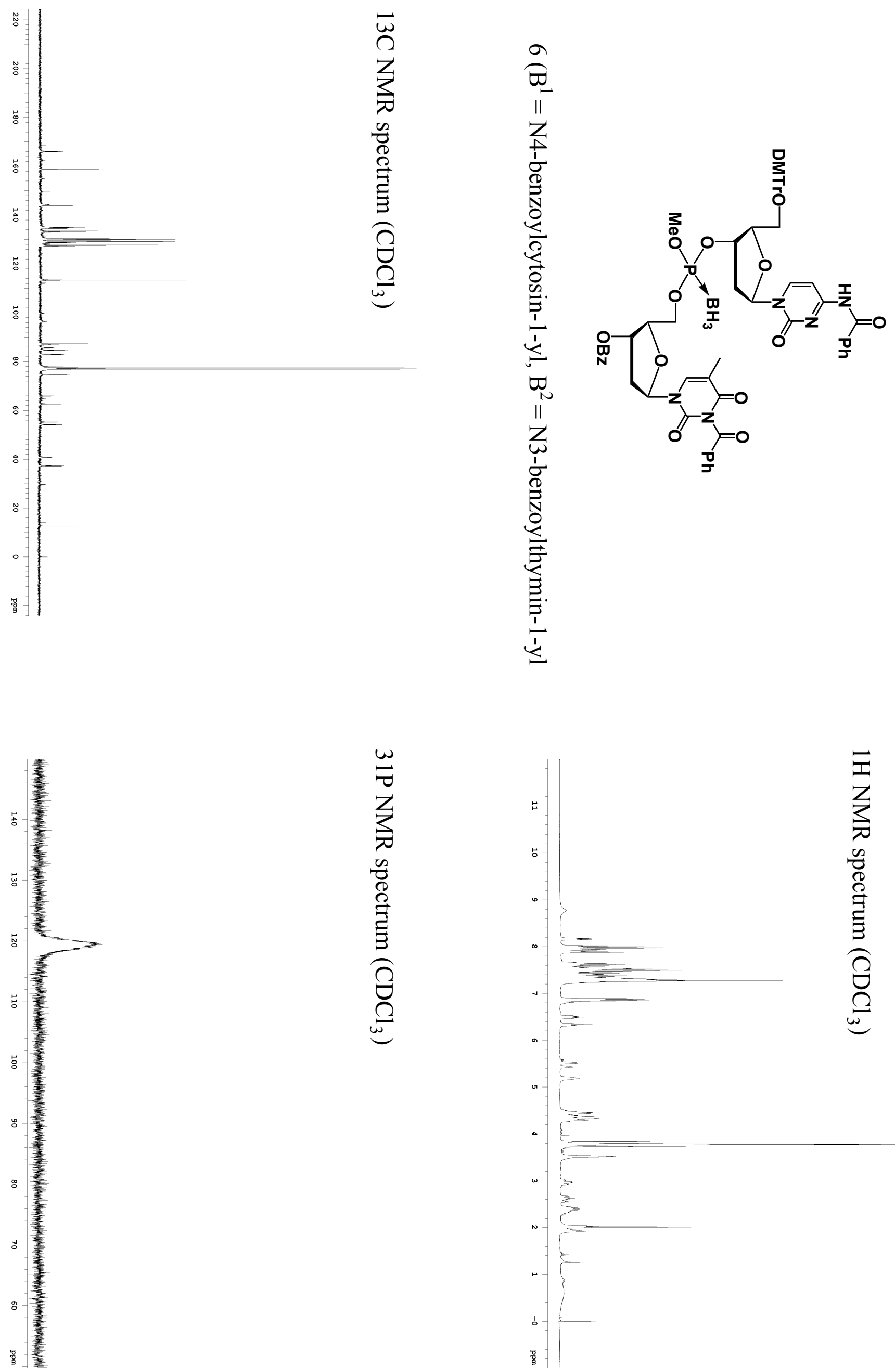

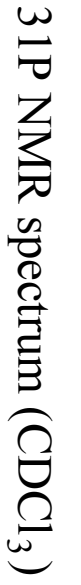

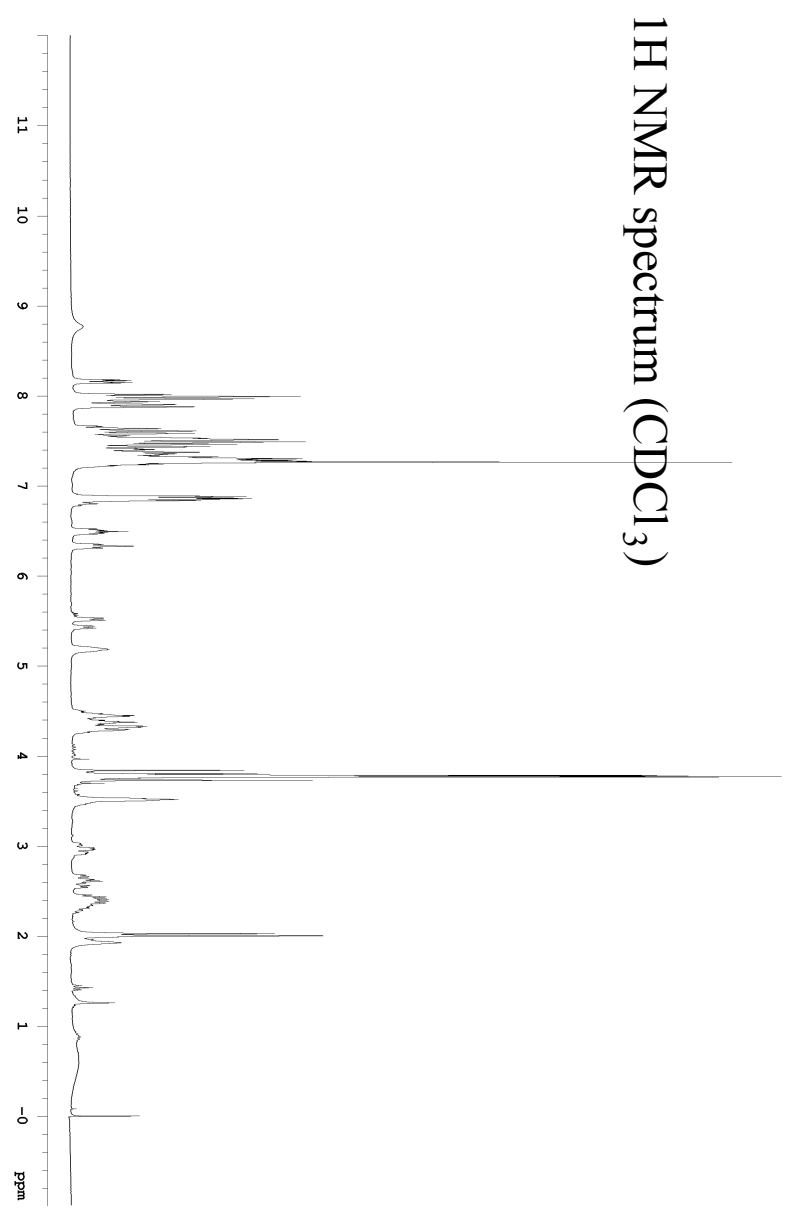


Supporting Info Page 27

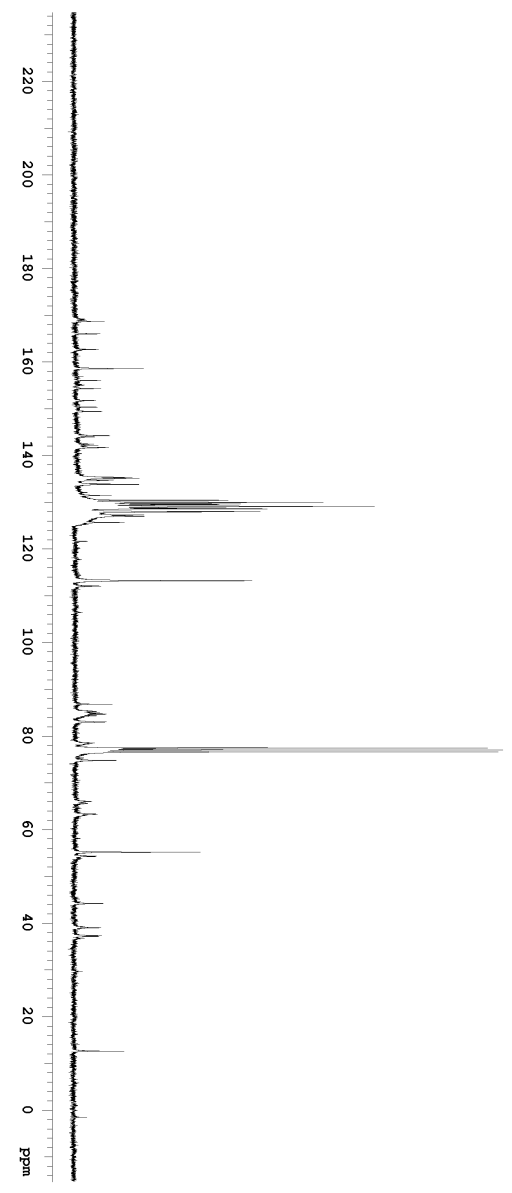

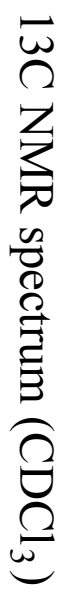

ㅁำ

岕

突

突这

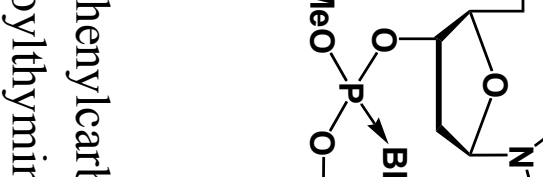

;

㐫芯

交

它

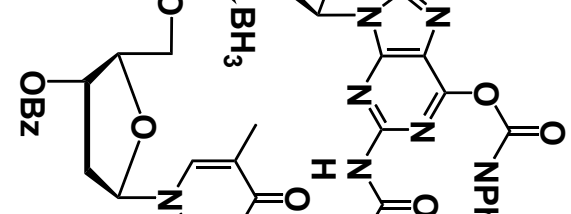

空

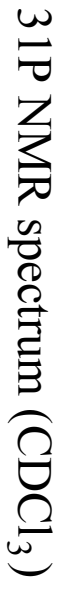


Supporting Info Page 28

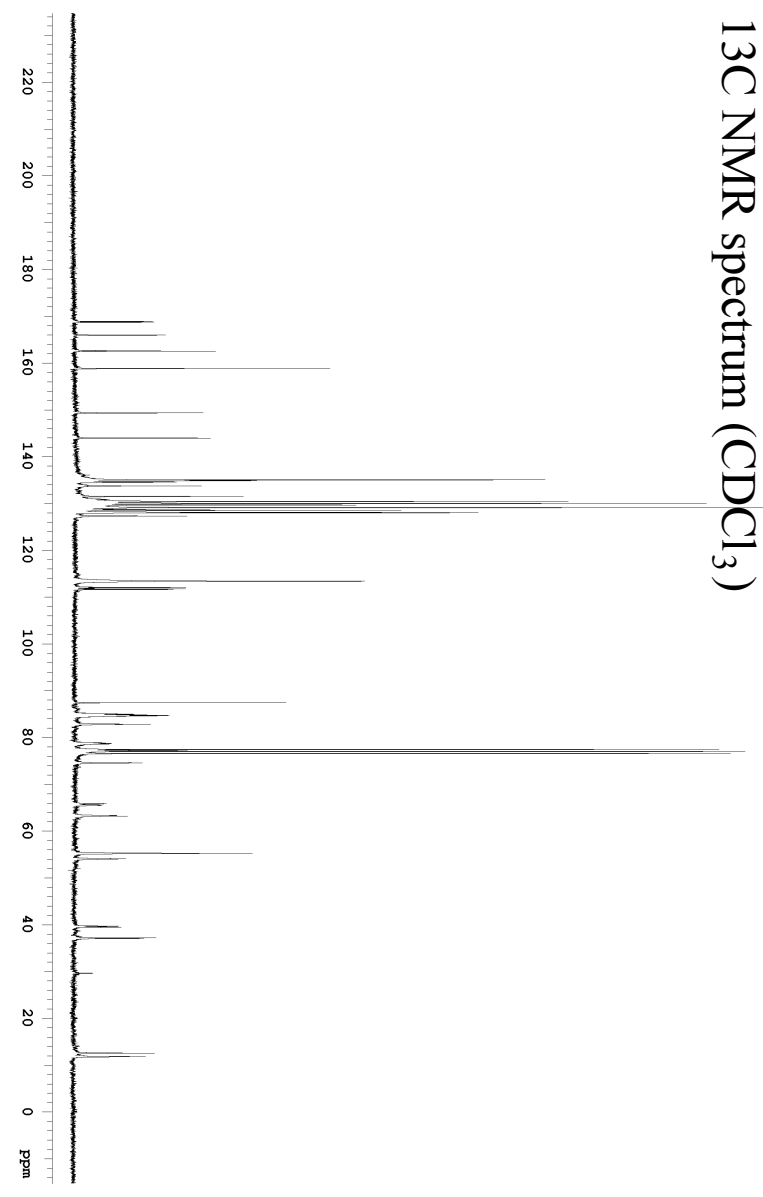

Ð્ટ
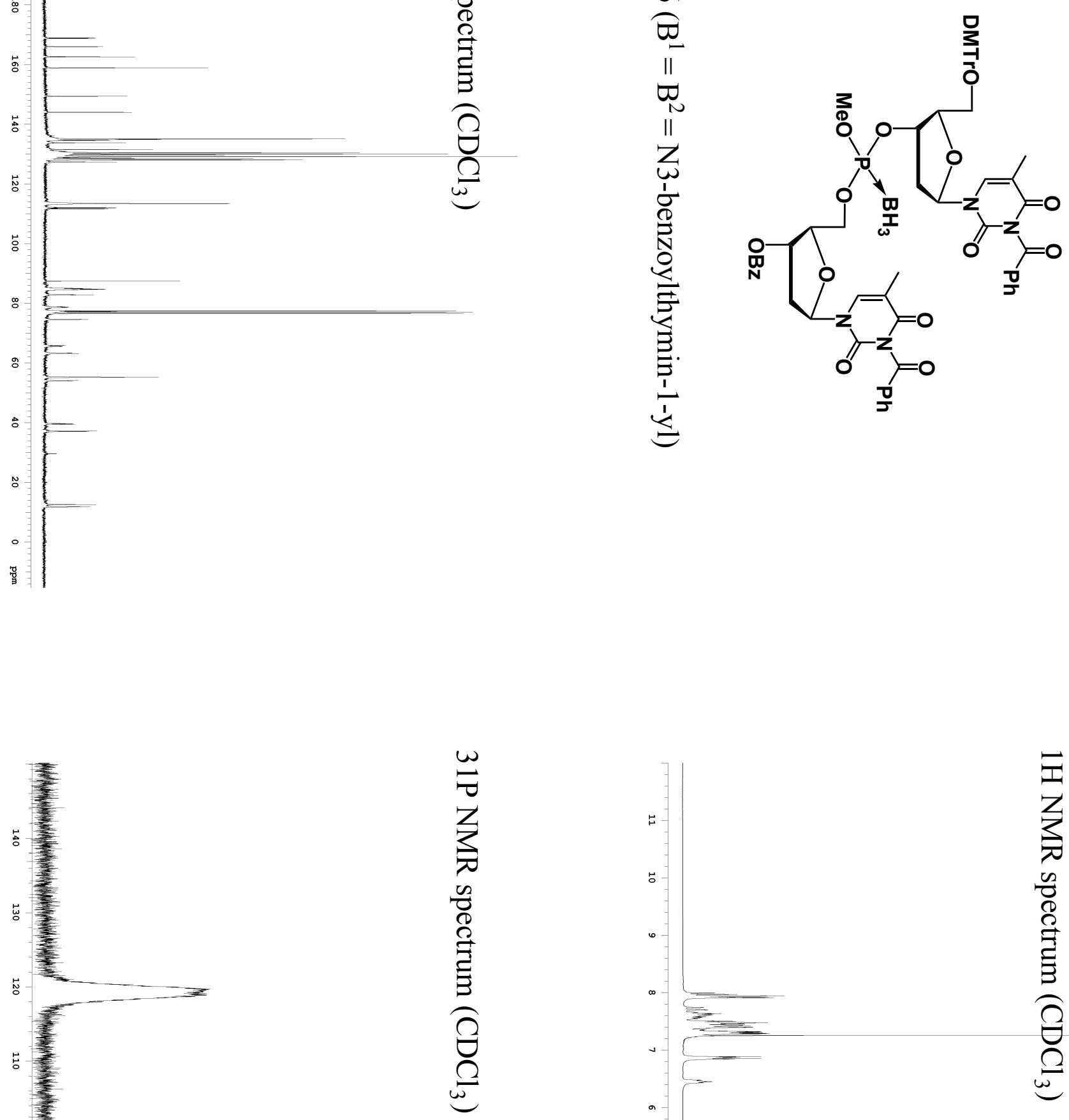

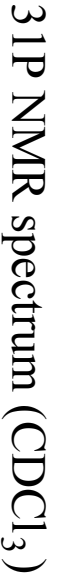

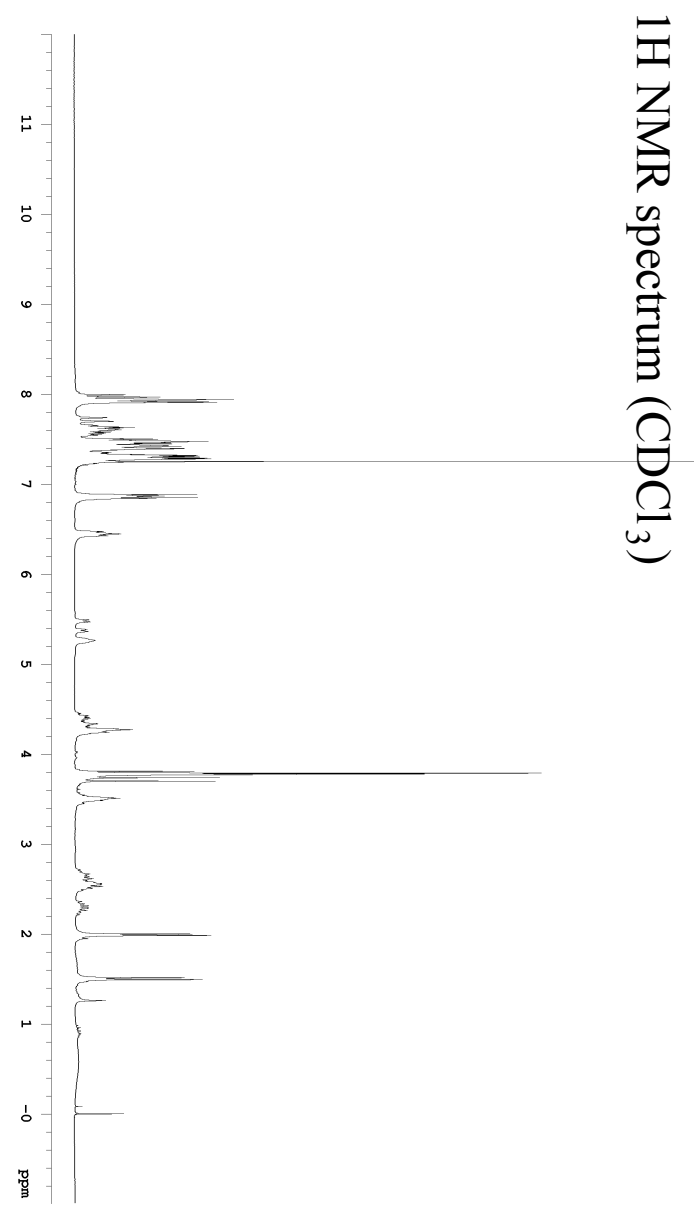


Supporting Info Page 29
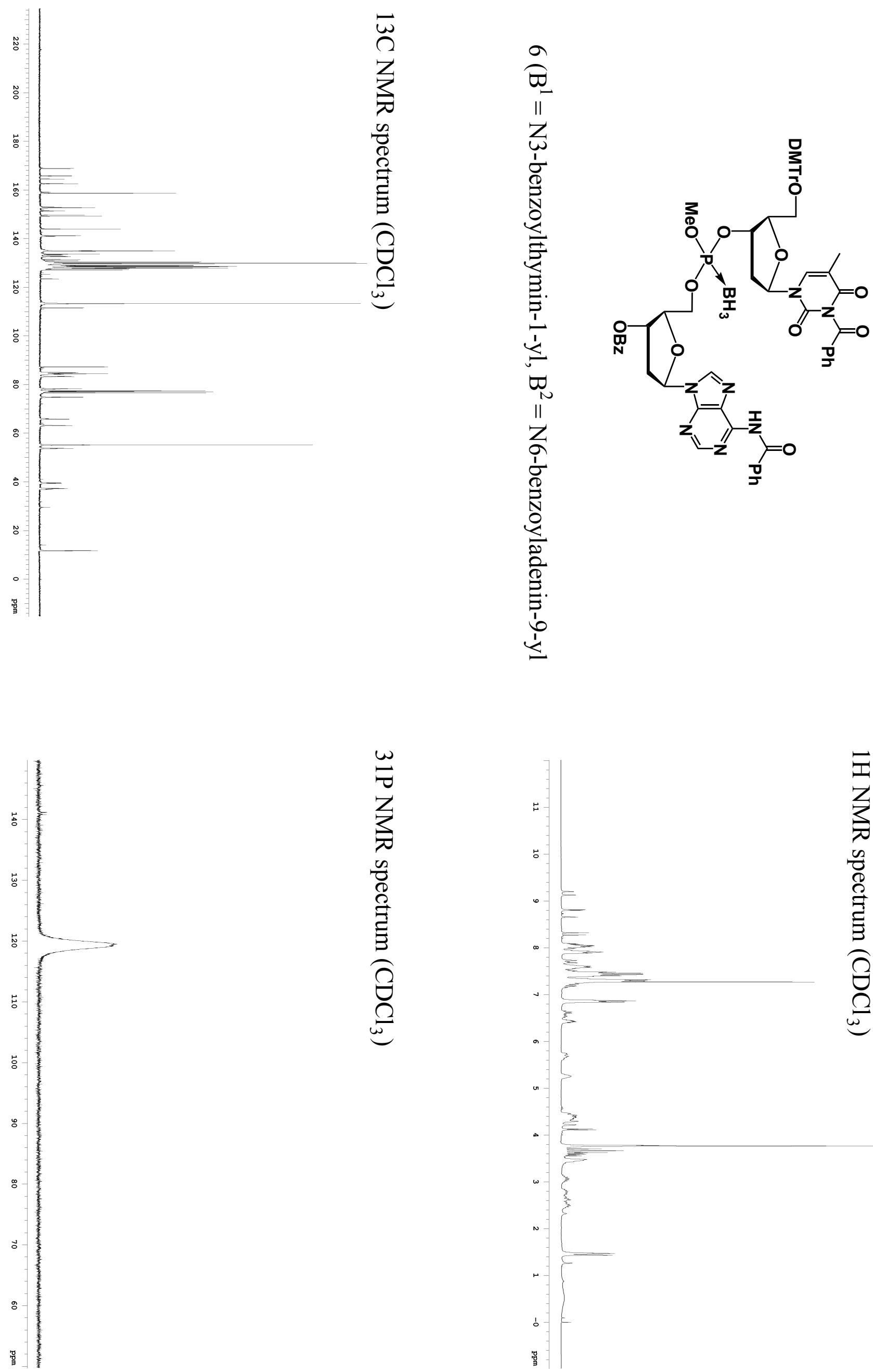

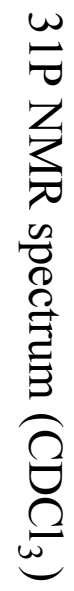


Supporting Info Page 30
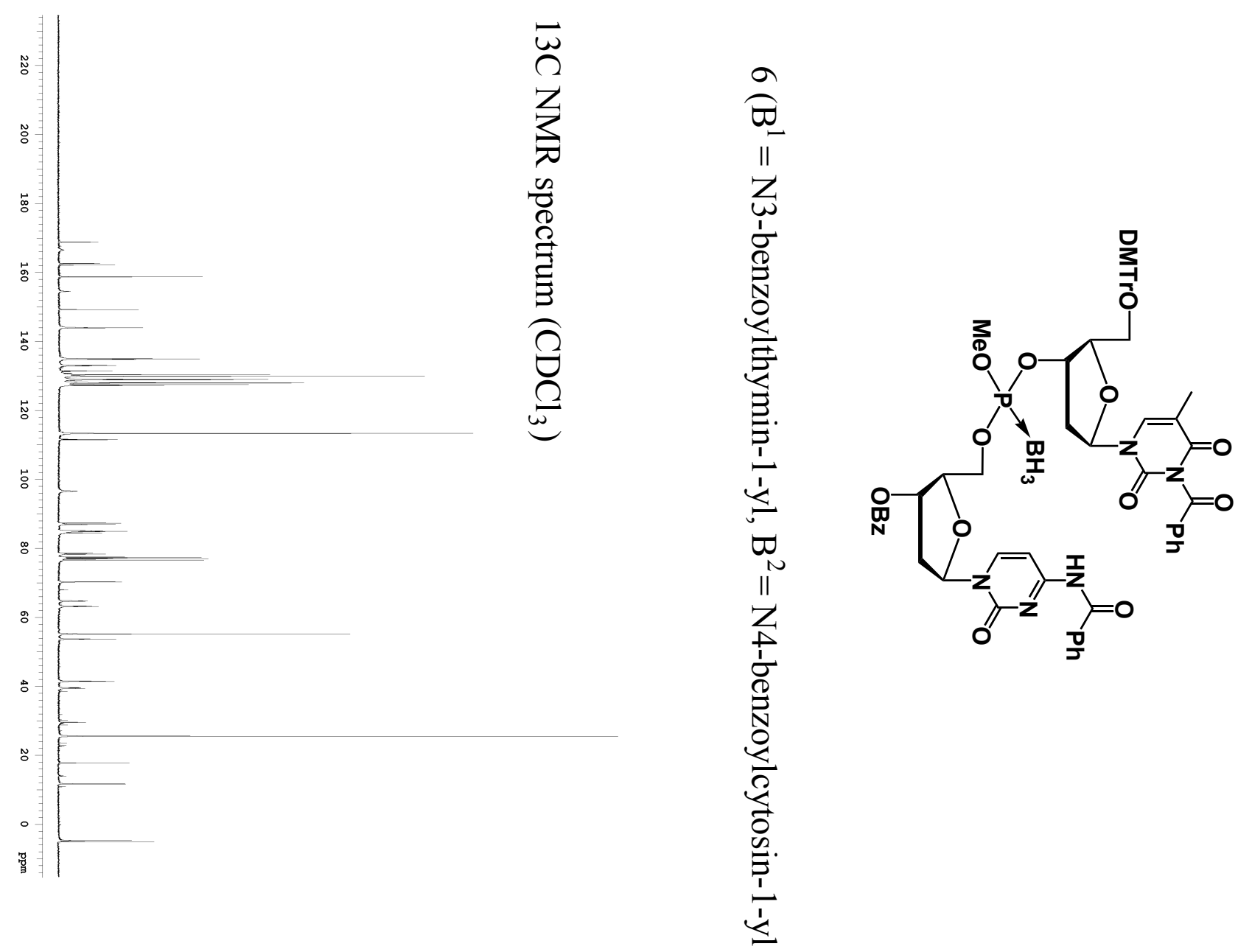

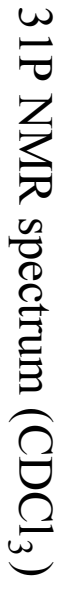

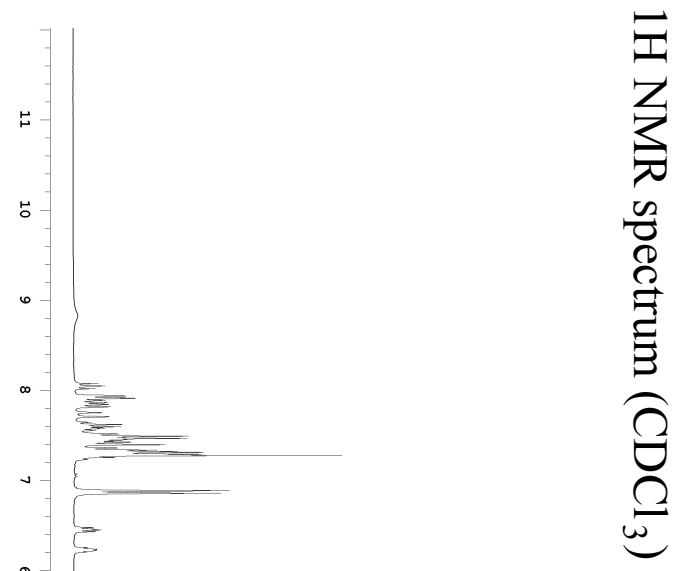


Supporting Info Page 31

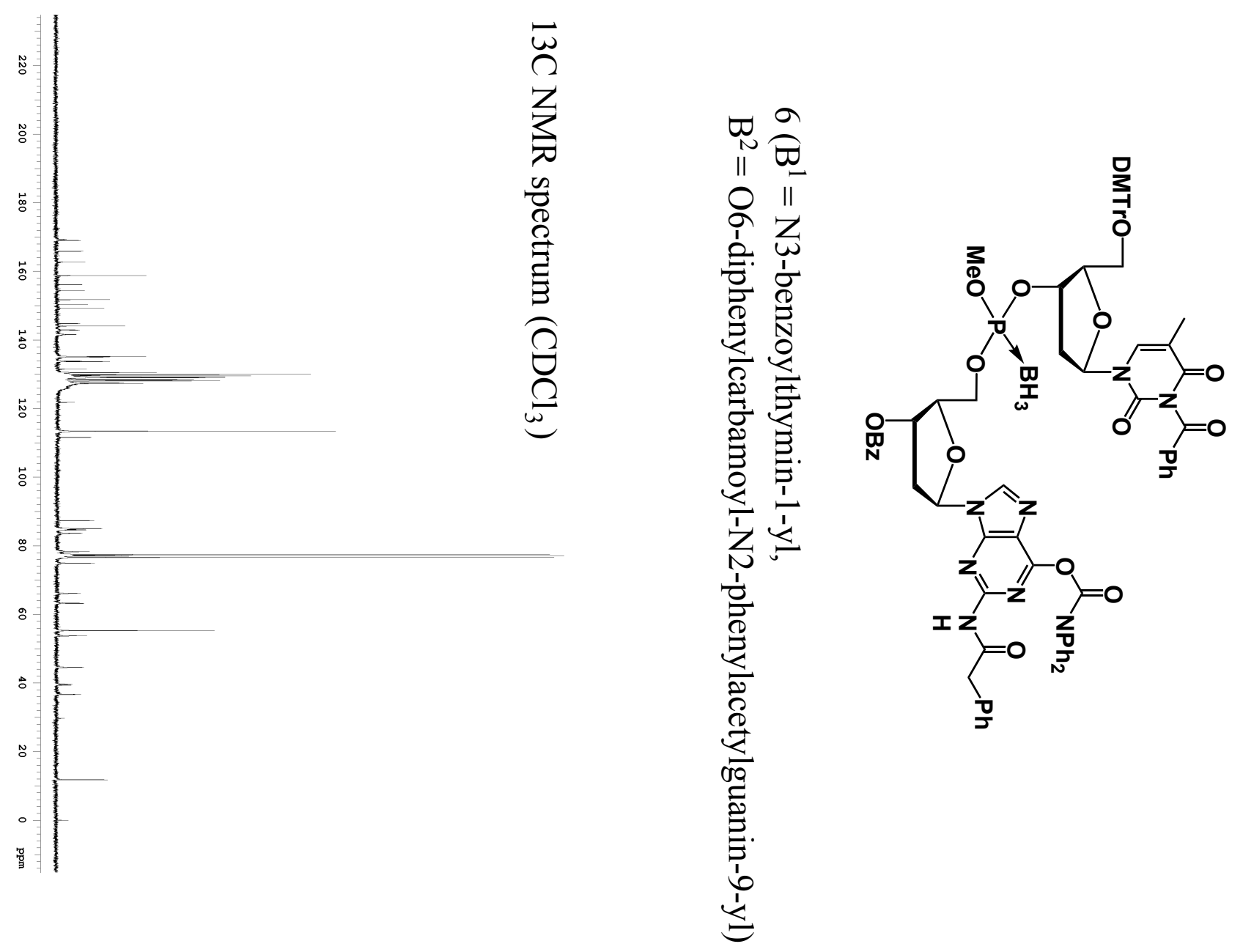

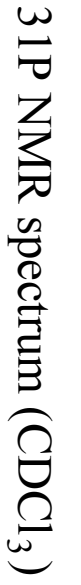

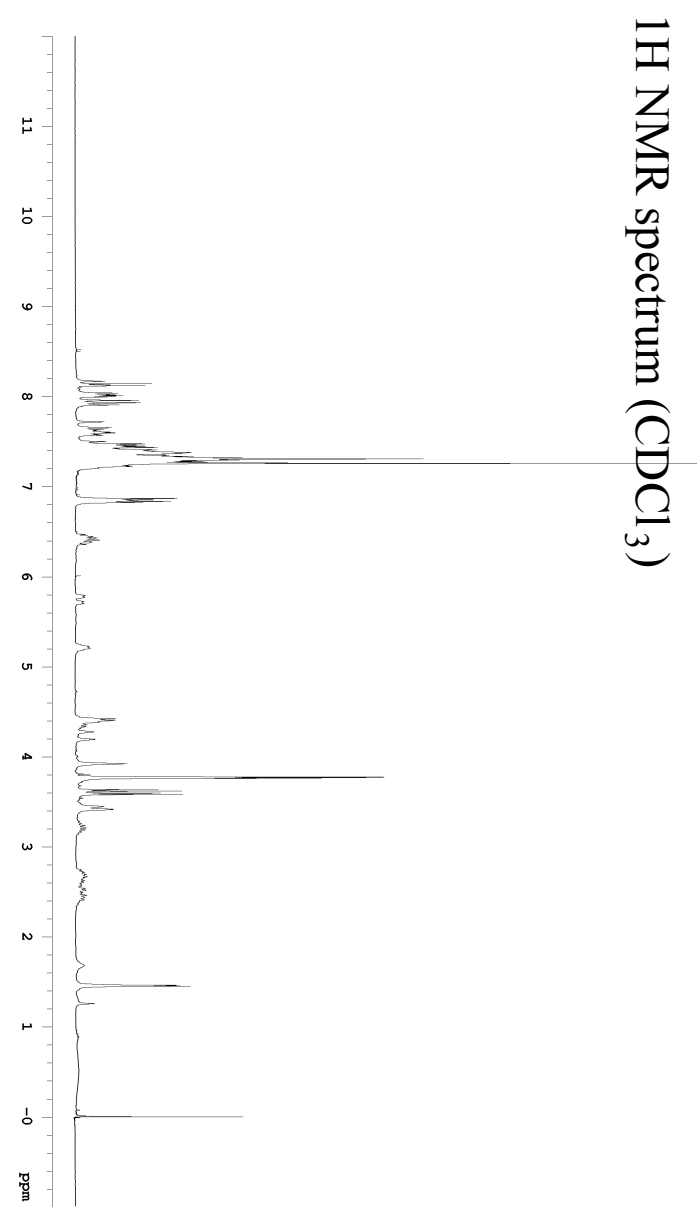


Supporting Info Page 32
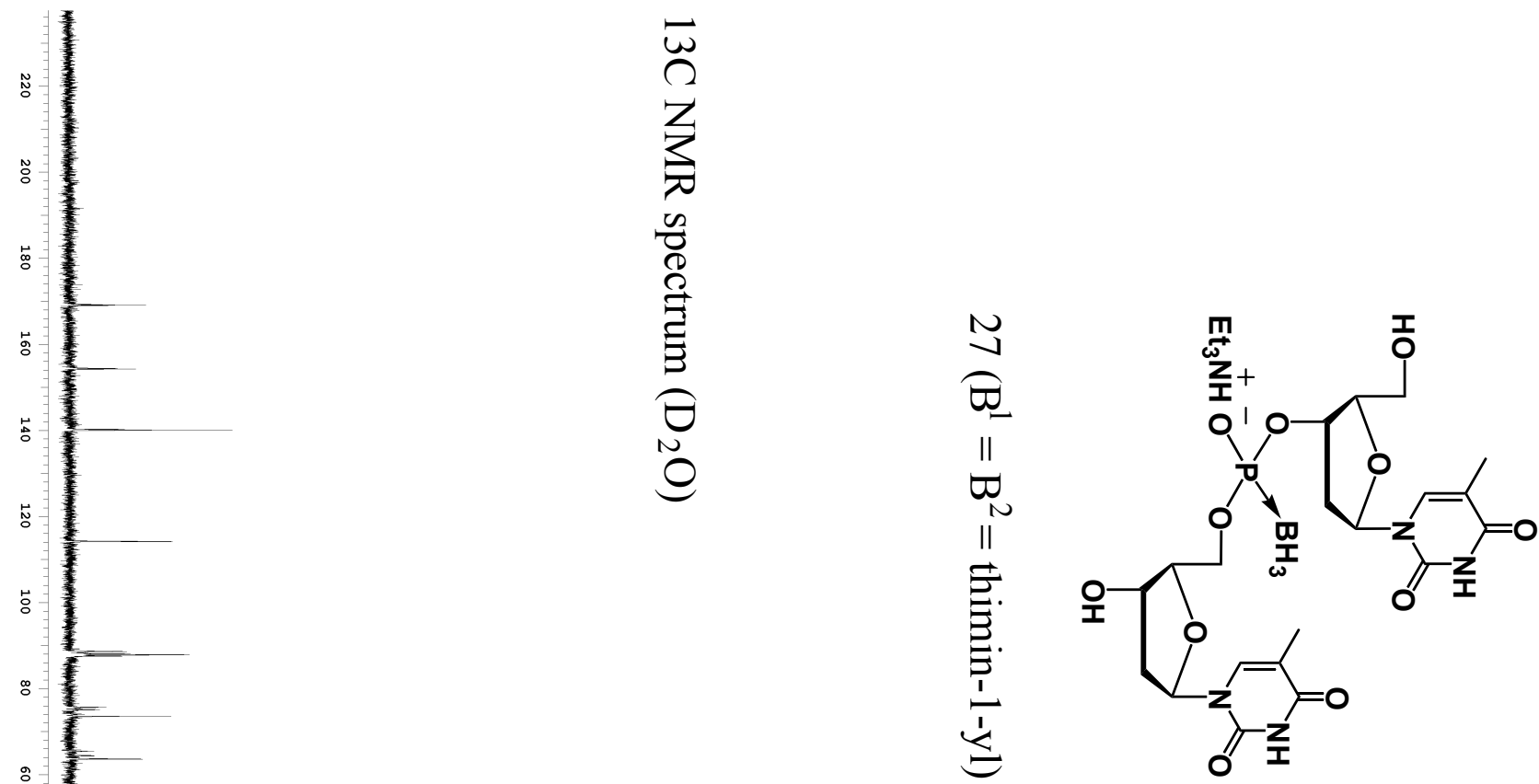

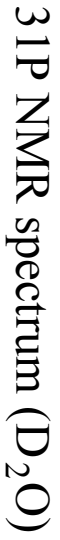

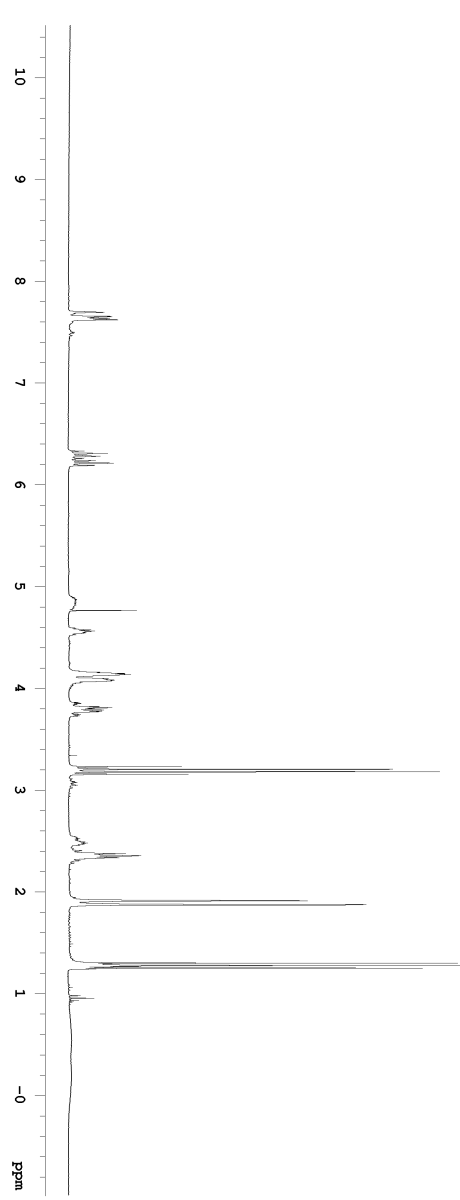

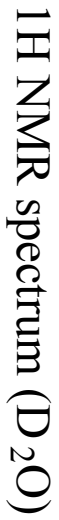
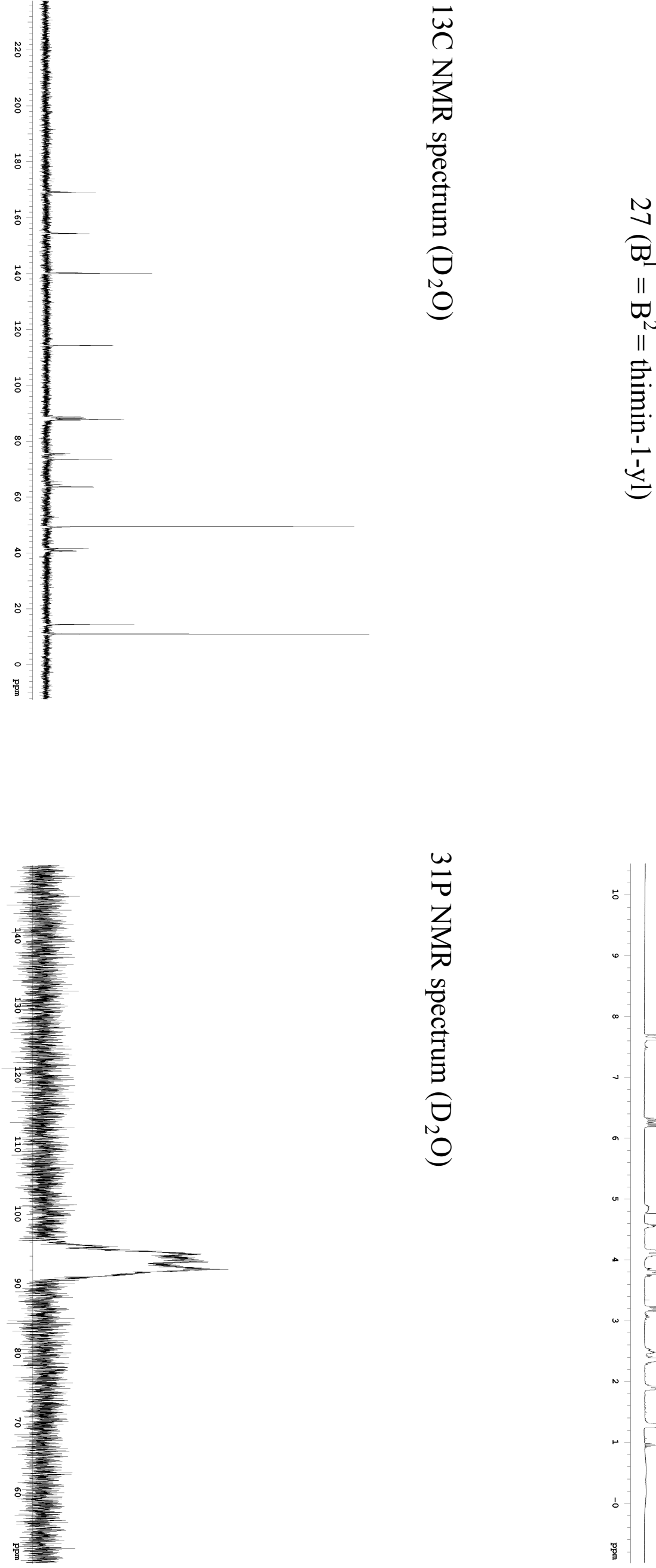
Suppoting Info Page 33

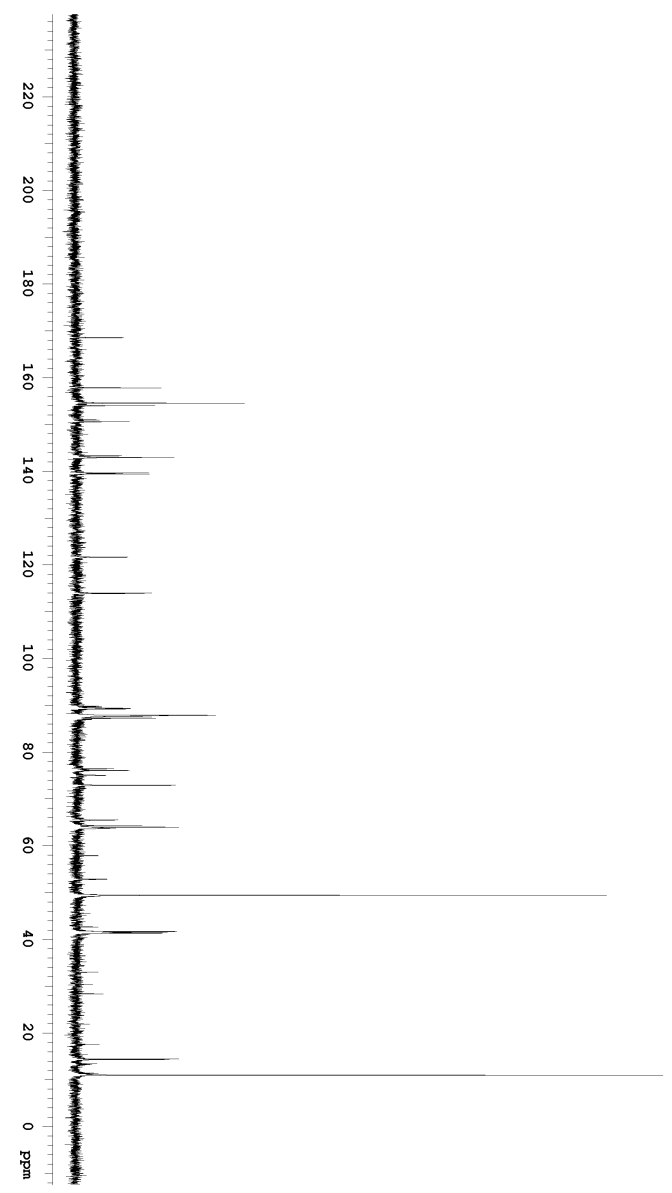

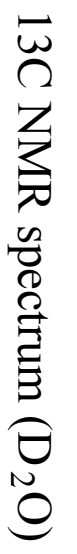

ڤิ)

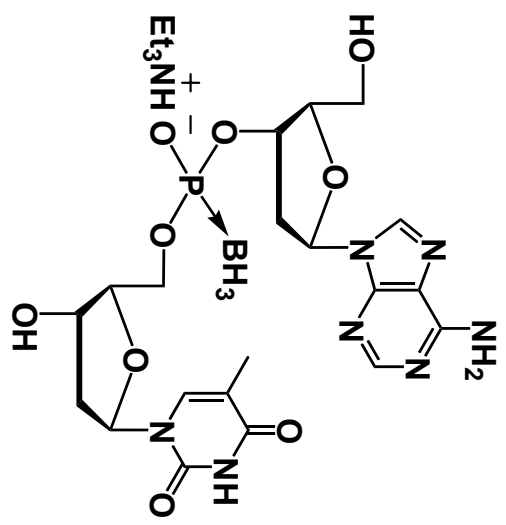

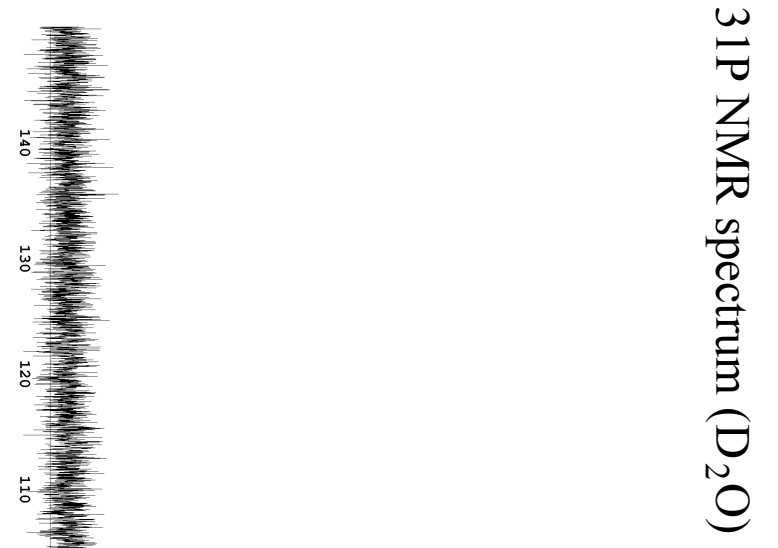

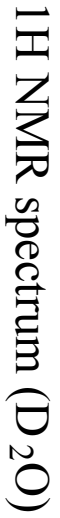
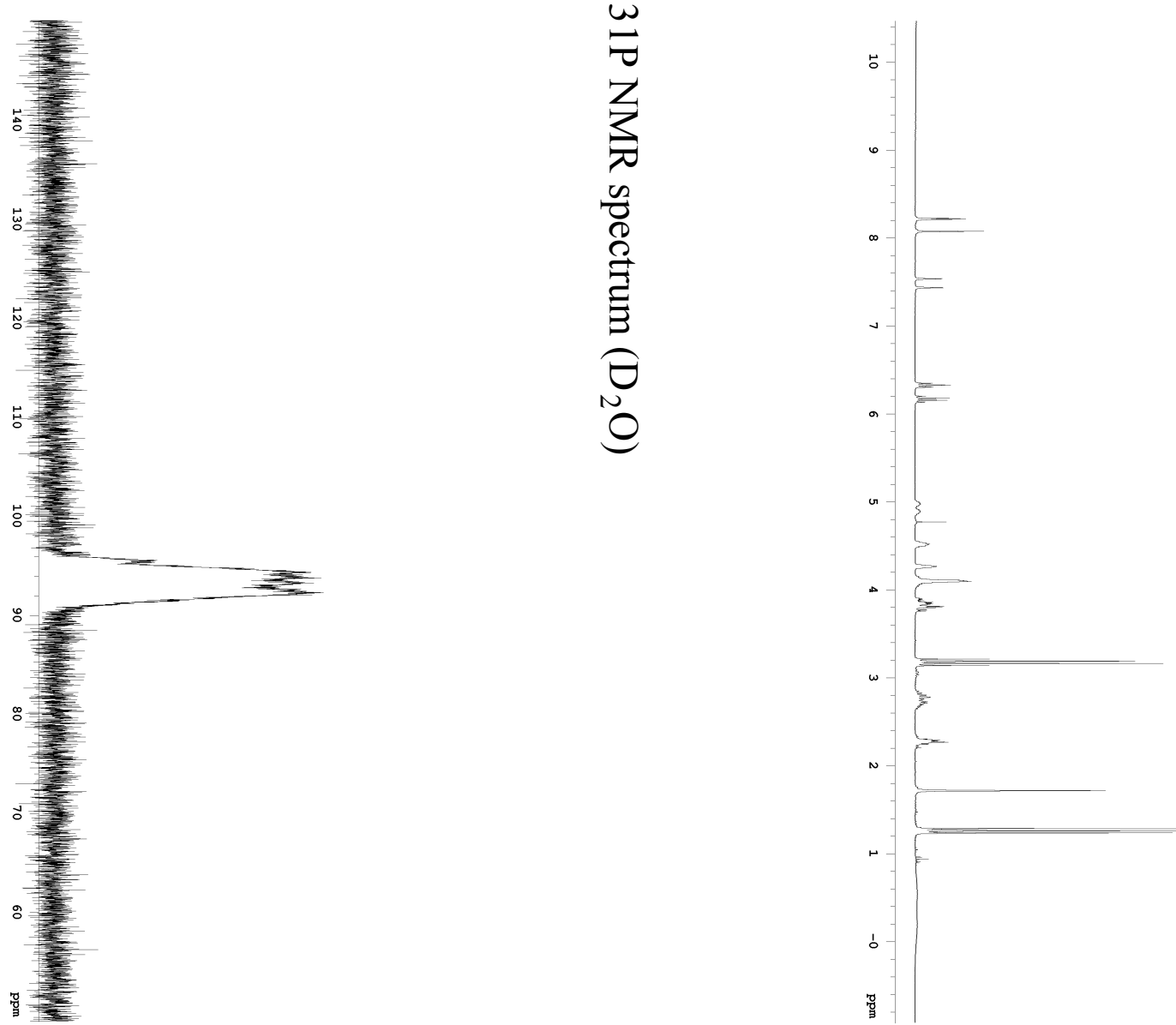
Supporting Info Page 34

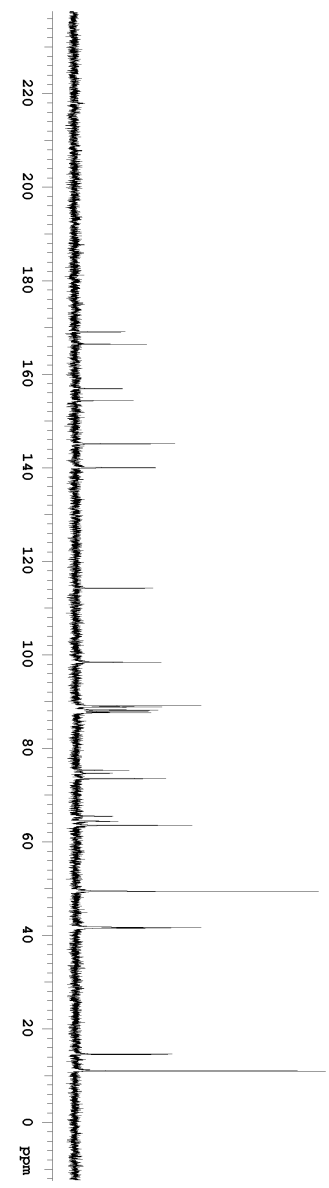

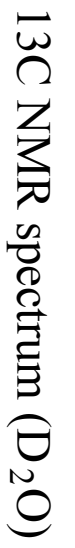
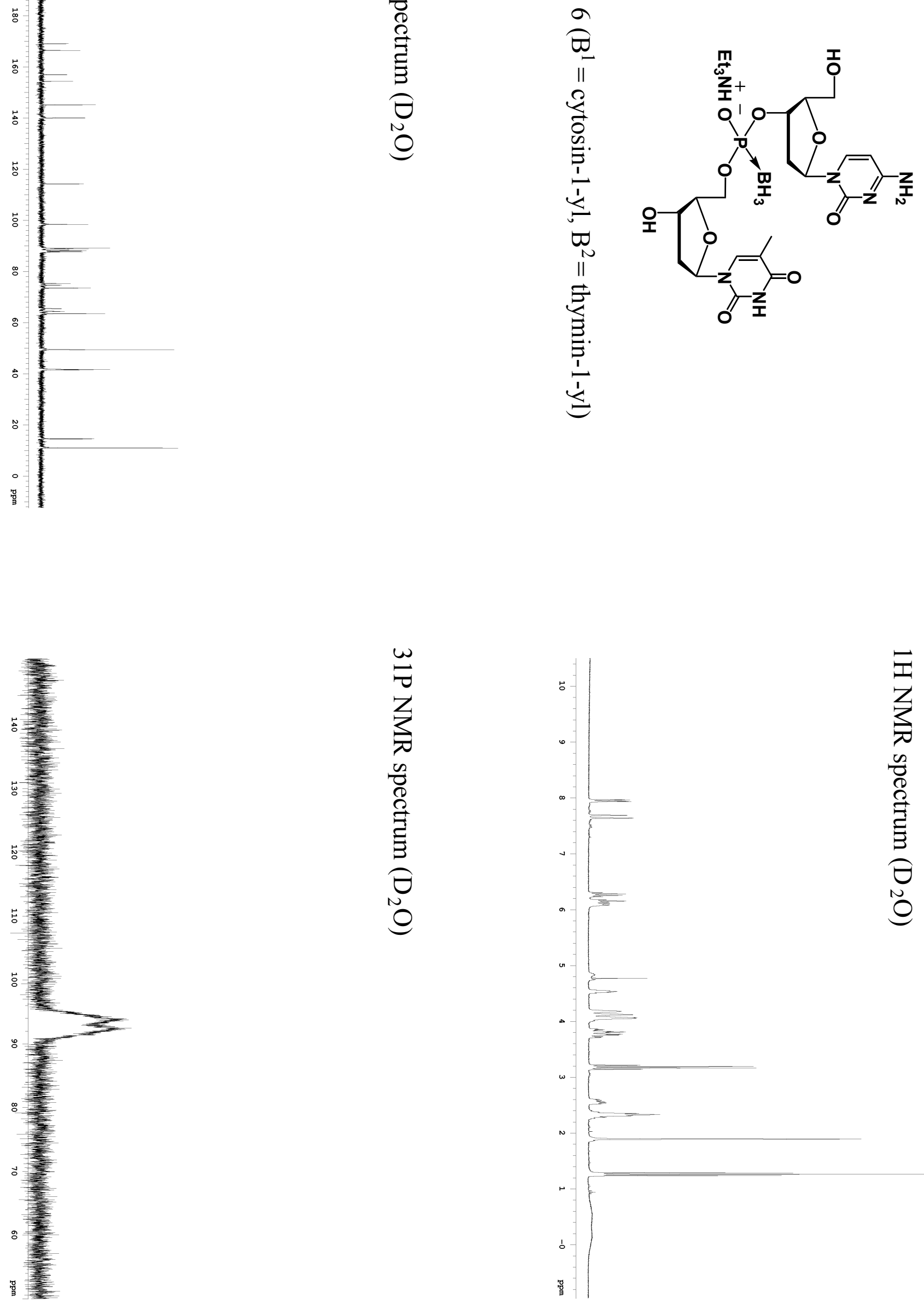

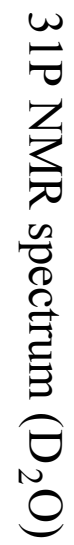

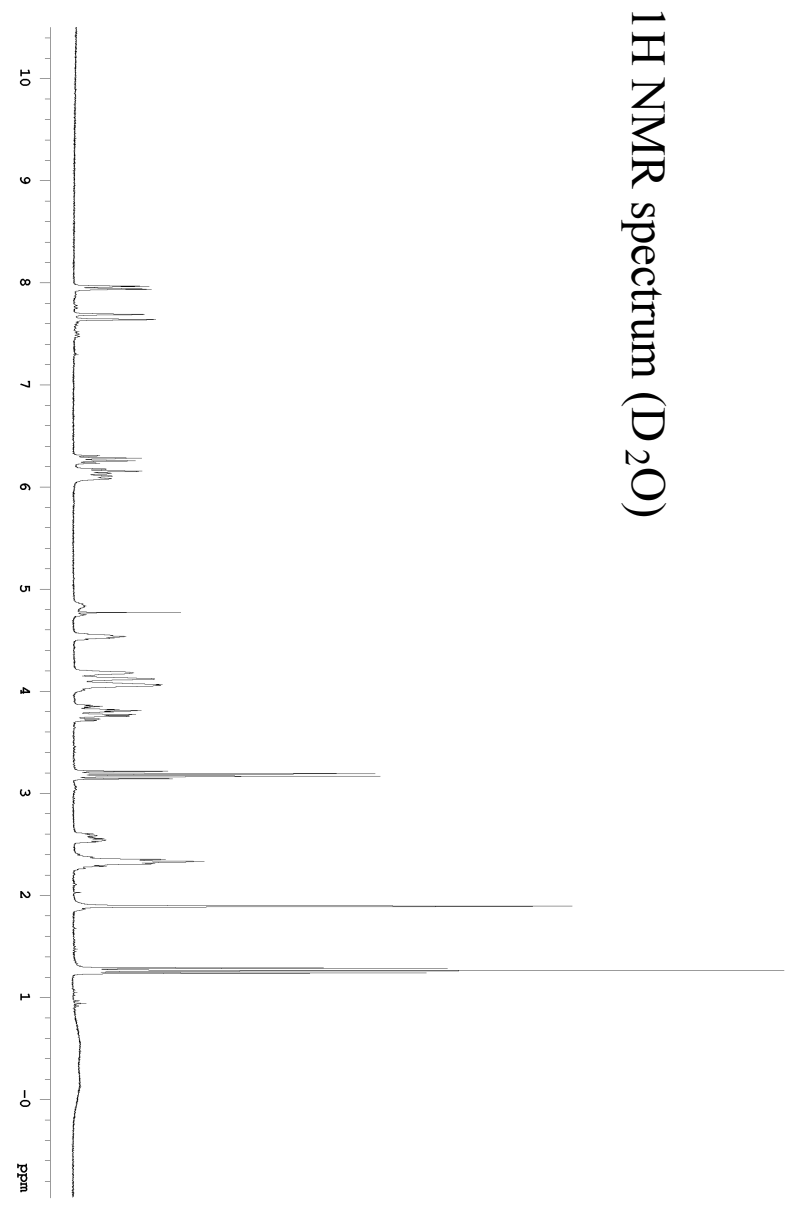


Suppoting Info Page 35
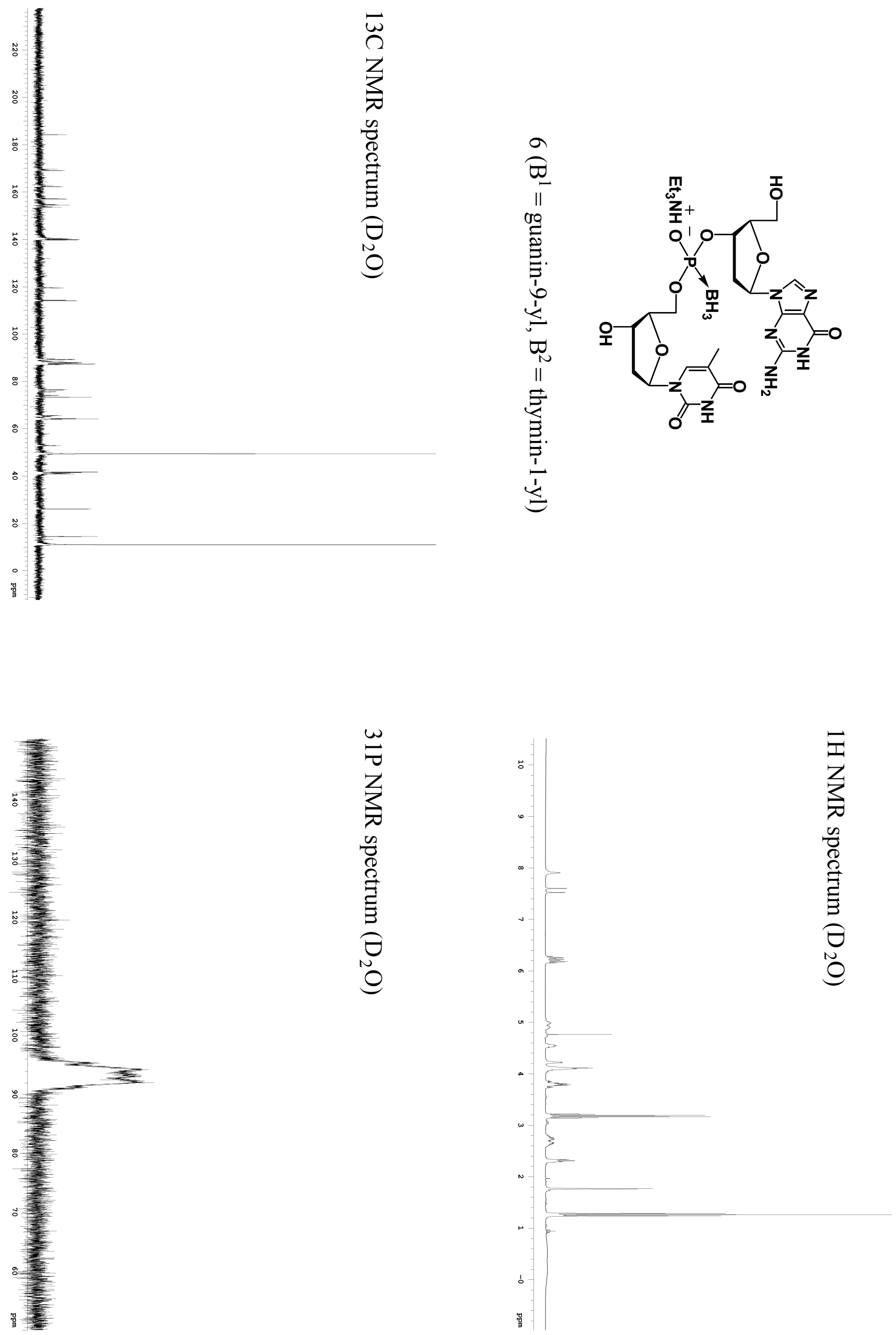